\title{
Using the thermal Gaussian approximation for the Boltzmann operator in semiclassical initial value time correlation functions
}

\author{
Jian Liu and William H. Miller \\ Department of Chemistry and K. S. Pitzer Center for Theoretical Chemistry \\ University of California, \\ and Chemical Science Division, Lawrence Berkeley National Laboratory \\ Berkeley, California 94720-1460
}

\begin{abstract}
The thermal Gaussian approximation (TGA) recently developed by Mandelshtam et al [Chem. Phys. Lett. 381, 117 (2003)] has been demonstrated to be a practical way for approximating the Boltzmann operator $\exp (-\beta \hat{H})$ for multidimensional systems. In this paper the TGA is combined with semiclassical (SC) initial value representations (IVRs) for thermal time correlation functions. Specifically, it is used with the linearized SC-IVR (LSC-IVR, equivalent to the classical Wigner model), and the 'forward-backward semiclassical dynamics' (FBSD) approximation developed by Makri et al. Use of the TGA with both of these approximate SC-IVRs allows the oscillatory part of the IVR to be integrated out explicitly, providing an extremely simple result that is readily applicable to large molecular systems. Calculation of the force-force autocorrelation for a strongly anharmonic oscillator demonstrates its accuracy, and of the velocity autocorrelation function (and thus the diffusion coefficient) of liquid neon demonstrates its applicability.
\end{abstract}




\section{Introduction}

There is currently a great deal of effort focused on developing ways ${ }^{1-7}$ for adding quantum mechanical effects to classical molecular dynamics (MD) simulations of chemical reactions, and other dynamical processes, in large molecular systems. Though purely classical MD simulations are adequate for many purposes, there is no doubt that quantum aspects of the dynamics will sometimes be important, and one will not know whether or not this is the case unless one has the ability of including them in the treatment, even if only approximately. Applications to a wide variety of molecular phenomena are obvious.

Semiclassical (SC) theory ${ }^{8-12}$ provides one way for adding quantum effects to classical MD simulations, and there is ample evidence that the SC approximation is a usefully accurate description of essentially all quantum effects in molecular dynamics ${ }^{2,7,13-18}$. For systems with many degrees of freedom, various initial value representations (IVRs) of SC theory provide the first step toward a practical way for carrying out SC calculations; this effectively replaces the non-linear boundary value problem of traditional SC theory with a Monte Carlo average over the initial conditions of classical trajectories ${ }^{9-12}$, a procedure much akin to what is done in classical MD simulations, allowing one to borrow from the great deal of computational development in that field. The added difficulty of an SC-IVR calculation, compared to a classical MD one, is the phase of the integrand, which carries all the quantum coherence information; thus all of the special techniques ${ }^{3-5,14,19,20}$ developed for carrying out SC-IVR calculations are concerned with this phase.

In this paper we focus of two of the simplest SC-IVR's, the 'linearized' approximation to the SCIVR (LSC-IVR) ${ }^{3,6,14}$, which yields the 'classical Wigner model', and Makri et al's simplified version of a forward-backward approximation to the SC-IVR, which they refer to as 'forward-backward semiclassical dynamics' (FBSD) ${ }^{4,5}$. Both of these approaches deal with the 'phase problem' by assuming that the two classical trajectories inherent to a time correlation function (see Section II) are close to one another. The only remaining issue is the quantum Boltzmann operator which appears in a thermal correlation function (see Section II). In the first applications of the LSC-IVR (to reactive flux correlation functions, and thus reaction rates) the Boltzmann operator was approximated as harmonic about the saddle point (transition state) on the potential surface ${ }^{3}$; this worked fine so long as the temperature was not too low. Geva et al later developed a more general 'local harmonic' approximation ${ }^{7}$ for the Boltzmann operator that allowed them to carry out very impressive LSC-IVR calculations for vibrational relaxation in liquids (involving force-force autocorrelation functions $)^{7,21}$. Similarly, Rossky et al have used a variationally optimized local harmonic approximation ${ }^{6}$ for the Boltzmann operator in carrying out LSC-IVR calculations, and Coker et al have extended this latter approach to also be able to describe electronically non-adiabatic dynamics ${ }^{22}$.

More recently, Mandelshtam et al have developed a very interesting 'thermal Gaussian approximation' (TGA) ${ }^{23,24}$ for the Boltzmann operator. It is itself a SC approximation, based on Heller's earlier 'frozen Gaussian' approximation ${ }^{25}$ (except that it involves imaginary time propagation); it is also a type of 'local harmonic' approximation, but one about the classically evolving trajectory (in imaginary time). Metiu et al applied such approaches earlier ${ }^{26}$, and more recently Pollak et al extended the TGA by showing how quantum corrections can be added to $\mathrm{it}^{27}$. Other imaginary time SC approximations for the Boltzmann operator that have been applied to thermal time correlation functions are an imaginary Herman-Kluk-type SC-IVR by Makri and Miller ${ }^{28}$, and the imaginary time Van Vleck SC-IVR of Zhao and Miller ${ }^{29}$. These latter two approaches provide more accurate approximations for the Boltzmann operator but are not as easy to implement as the TGA.

The purpose of this paper is to use the TGA of Mandelshtam et al within the LSC-IVR and FBSD approximations for thermal time correlation functions, to see how well it works and to demonstrate its potential for application to large molecular systems. Section II describes the SC-IVR theory of time correlation functions, including the LSC-IVR and the FBSD methods. A brief review of the TGA is given in section III. Combinations of the TGA with the SC-IVR methods (TGA-LSC-IVR and TGA-FBSD) and their numerical advantages are discussed in section IV. Several numerical applications of the TGA-LSC- 
IVR and TGA-FBSD methods are presented in section V, including a strong anharmonic one-dimensional model system and a complex system (liquid neon). Finally, some concluding remarks appear in section VI.

\section{SC-IVR Calculation of Time Correlation Functions}

Most quantities of interest in the dynamics of complex systems can be expressed in terms of time correlation functions $^{30}$. For example, dipole moment correlation functions are related to absorption spectra, flux correlation functions yield reaction rates, velocity correlation functions can be used to calculate diffusion constants, and vibrational energy relaxation rate constants can be expressed in terms of force correlation functions. The standard real time correlation function is of the form

$$
C_{A B}(t)=\frac{1}{Z} \operatorname{Tr}\left(e^{-\beta \hat{H}} \hat{A} e^{i \hat{H} t / \hbar} \hat{B} e^{-i \hat{H} t / \hbar}\right)=\operatorname{Tr}\left(\hat{\rho}_{0} \hat{A} e^{i \hat{H} t / \hbar} \hat{B} e^{-i \hat{H} t / \hbar}\right)
$$

or sometimes it is convenient to use the following symmetrized version ${ }^{31}$

$$
C_{A B}(t)=\frac{1}{Z} \operatorname{Tr}\left(e^{-\beta \hat{H} / 2} \hat{A} e^{-\beta \hat{H} / 2} e^{i \hat{H} t / \hbar} \hat{B} e^{-i \hat{H} t / \hbar}\right)
$$

Here $\hat{H}$ is the (time-independent) Hamiltonian for the system, which for large molecular systems is usually expressed in terms of its Cartesian coordinates and momenta

$$
H=\frac{1}{2} \hat{\mathbf{p}}^{\mathrm{T}} \mathbf{M}^{-1} \hat{\mathbf{p}}+V(\hat{\mathbf{q}})=\hat{H}_{0}+V(\hat{\mathbf{q}})
$$

where $\mathbf{M}$ is the (diagonal) mass matrix and $\hat{\mathbf{p}}, \hat{\mathbf{q}}$ are the momentum and coordinate operators, respectively. Also, in Eq 2.1 and 2.2, $Z=\operatorname{Tr} e^{-\beta \hat{H}}\left(\beta=1 / k_{B} T\right)$ is the partition function, $\hat{\rho}_{0}=e^{-\beta \hat{H}} / Z$ is the equilibrium density operator, and $\hat{A}$ and $\hat{B}$ are operators relevant to the specific property of interest.

The SC-IVR approximates the time evolution operator $e^{-i \hat{H} t / \hbar}$ by a phase space average over the initial conditions of classical trajectories ${ }^{9,10,32,33}$. The original, Van Vleck version of the IVR is

$$
e^{-i \hat{H} t / \hbar}=\int d \mathbf{p}_{0} \int d \mathbf{q}_{0} \sqrt{M_{q p} /(2 \pi i \hbar)^{3 N}} e^{i S_{t}\left(\mathbf{p}_{0}, \mathbf{q}_{0}\right) / \hbar}\left|\mathbf{q}_{t}\right\rangle\left\langle\mathbf{q}_{0}\right|
$$

where $\left(\mathbf{q}_{0}, \mathbf{p}_{0}\right)$ is the set of initial conditions (i.e., coordinates and momenta) for a classical trajectory, $\left(\mathbf{p}_{t}\left(\mathbf{q}_{0}, \mathbf{p}_{0}\right), \mathbf{q}_{t}\left(\mathbf{q}_{0}, \mathbf{p}_{0}\right)\right)$ the phase point at time $t$ which evolves from that trajectory, $S_{t}\left(\mathbf{q}_{0}, \mathbf{p}_{0}\right)$ the classical action along it, and $M_{q p}$ the determinant of the Jacobian matrix relating the final position and initial momentum,

$$
M_{q p}=\operatorname{det}\left(\partial \mathbf{q}_{t}\left(\mathbf{q}_{0}, \mathbf{p}_{0}\right) / \partial \mathbf{p}_{0}\right)
$$

For the correlation function in Eq. (2.1) or (2.2), one needs to insert two such representations of the evolution propagator, yielding the following double phase space average for the correlation function, 


$$
\begin{aligned}
C_{A B}(t) & =(2 \pi \hbar)^{-3 N} Z^{-1} \int d \mathbf{p}_{0} \int d \mathbf{q}_{0} \int d \mathbf{p}_{0}^{\prime} \int d \mathbf{q}_{0}^{\prime}\left(M_{q p} M_{q p}^{\prime}\right)^{1 / 2}\left\langle\mathbf{q}_{0}\left|\hat{A}^{\beta}\right| \mathbf{q}_{0}^{\prime}\right\rangle \\
& \cdot e^{i S_{t}\left(\mathbf{p}_{0}, \mathbf{q}_{0}\right) / \hbar} e^{-i S_{t}\left(\mathbf{p}_{0}^{\prime}, \mathbf{q}_{0}^{\prime}\right) / \hbar}\left\langle\mathbf{q}_{t}^{\prime}|\hat{B}| \mathbf{q}_{t}\right\rangle
\end{aligned}
$$

where $\hat{A}^{\beta}=e^{-\beta \hat{H}} \hat{A}$ for Eq. (2.1) or $\hat{A}^{\beta}=e^{-\beta \hat{H} / 2} \hat{A} e^{-\beta \hat{H} / 2}$ for Eq. (2.2). The primary difficulty in evaluating this expression is the oscillatory character coming from the difference between the action integrals of the trajectories with initial conditions $\left(\mathbf{q}_{0}, \mathbf{p}_{0}\right)$ and $\left(\mathbf{q}_{0}^{\prime}, \mathbf{p}_{0}^{\prime}\right)$.

One way to deal with this 'phase problem', proposed by Miller and coworkers ${ }^{3,14}$, is to make the (rather drastic) approximation of assuming that the dominant contribution to the double phase space average comes from phase points $\left(\mathbf{q}_{0}, \mathbf{p}_{0}\right)$ and $\left(\mathbf{q}_{0}^{\prime}, \mathbf{p}_{0}^{\prime}\right)$ that are close to one another. Changing to sum and difference variables,

$$
\begin{aligned}
& \overline{\mathbf{p}}_{0}=\frac{1}{2}\left(\mathbf{p}_{0}+\mathbf{p}_{0}^{\prime}\right), \quad \overline{\mathbf{q}}_{0}=\frac{1}{2}\left(\mathbf{q}_{0}+\mathbf{q}_{0}^{\prime}\right), \\
& \ddot{\mathbf{A}} \mathbf{p}_{0}=\mathbf{p}_{0}-\mathbf{p}^{\prime}, \quad \ddot{\mathbf{A}} \mathbf{q}_{0}=\mathbf{q}_{0}-\mathbf{q}_{0}^{\prime}
\end{aligned}
$$

and expanding all quantities in the integrand of Eq. (2.6) to first order in $\ddot{A} \mathbf{p}_{0}$ and $\ddot{\mathbf{A q}} \mathbf{q}_{0}$, gives the linearized SC-IVR (LSC-IVR), or classical Wigner model for the correlation function,

$$
C_{A B}^{L S C-I V R}(t)=Z^{-1} \int d \mathbf{p}_{0} \int d \mathbf{q}_{0} A_{w}^{\beta}\left(\mathbf{q}_{0}, \mathbf{p}_{0}\right) B_{w}\left(\mathbf{q}_{t}, \mathbf{p}_{t}\right)
$$

where here $\left(\mathbf{q}_{0}, \mathbf{p}_{0}\right) \equiv\left(\overline{\mathbf{q}}_{0}, \overline{\mathbf{p}}_{0}\right) \quad$ (i.e., the 'bars' have been removed), and $A_{w}^{\beta}$ and $B_{w}$ are the Wigner functions corresponding to these operators,

$$
O_{w}(\mathbf{q}, \mathbf{p})=(2 \pi \hbar)^{-3 N} \int d \Delta \mathbf{q}\langle\mathbf{q}-\Delta \mathbf{q} / 2|\hat{O}| \mathbf{q}+\Delta \mathbf{q} / 2\rangle e^{i \mathbf{p}^{T} \ddot{A} \mathbf{q} / \hbar}
$$

for any operator $\hat{O}$. I.e., the integrals over $\ddot{\mathbf{A}} \mathbf{p}_{0}$ and $\ddot{\mathbf{A}} \mathbf{q}_{0}$ have become the two Fourier integrals that produce the Wigner functions of the two operators. Eq. (2.8), with the remaining (single) phase space average, now has the form of the classical correlation function, the only difference being that the Wigner functions corresponding to operators $\hat{A}$ and $\hat{B}$ appear rather than the classical functions. The LSC-IVR result in Eq (2.9), also termed the 'classical Wigner' model, has been obtained by a variety of formulations, so the result itself is not new. What is interesting, though, is to realize that it is contained with the overall SC-IVR description, as a well-defined approximation to it.

Calculation of the Wigner function for operator $\hat{B}$ in Eq 2.8 is usually straight-forward; in fact, $\hat{B}$ is often a function only of coordinates or only of momenta, in which case its Wigner functions is simply the classical function itself. Calculating the Wigner function for operator $\hat{A}^{\beta}$, however, involves the Boltzmann operator with the total Hamiltonian of the complete system, so that carrying out the multidimensional Fourier transform to obtain it is far from trivial. Furthermore, it is necessary to do this in order obtain the distribution of initial conditions of momenta $\mathbf{p}_{0}$ for the real time trajectories. A rigorous way to treat the Boltzmaan operator is via a Feynman path integral expansion, but it is then in general not possible to evaluate the multidimensional Fourier transform explicitly to obtain the Wigner function for $\hat{A}^{\beta}$ (and thus the distribution of initial conditions of the momenta $\mathbf{p}_{0}$; Appendix A discusses and analyzes this situation in more detail. The inability to calculate the Wigner function of $\hat{A}^{\beta}$ exactly is 
in fact the reason for the various harmonic and local harmonic approximations to the Boltzmann operator noted above, and the TGA discussed below in Section III.

Another SC-IVR approach for the time evolution operators $e^{-i \hat{H} t / \hbar}$ is the Herman-Kluk, or coherent state IVR ${ }^{12}$

$$
e^{-i \hat{H} t / \hbar}=(2 \pi \hbar)^{-3 N} \int d \mathbf{q}_{0} \int d \mathbf{p}_{0} C_{t}\left(\mathbf{q}_{0}, \mathbf{p}_{0}\right) e^{i S_{t}\left(\mathbf{p}_{0}, \mathbf{q}_{0}\right) / \hbar}\left|\mathbf{q}_{t}, \mathbf{p}_{t}\right\rangle\left\langle\mathbf{q}_{0}, \mathbf{p}_{0}\right|
$$

where the pre-exponential factor is given by

$$
C_{t}\left(\mathbf{q}_{0}, \mathbf{p}_{0}\right)=2^{-3 N / 2}\left[\operatorname{det}\left(\tilde{\mathbf{A}}^{1 / 2} \frac{\partial \mathbf{q}_{t}}{\partial \mathbf{q}_{0}} \tilde{\mathbf{A}}^{-1 / 2}+\tilde{\mathbf{A}}^{-1 / 2} \frac{\partial \mathbf{p}_{t}}{\partial \mathbf{p}_{0}} \tilde{\mathbf{A}}^{1 / 2}-2 i \hbar \tilde{\mathbf{A}}^{1 / 2} \frac{\partial \mathbf{q}_{t}}{\partial \mathbf{p}_{0}} \tilde{\mathbf{A}}^{1 / 2}-\frac{1}{2 i \hbar} \tilde{\mathbf{A}}^{-1 / 2} \frac{\partial \mathbf{p}_{t}}{\partial \mathbf{q}_{0}} \tilde{\mathbf{A}}^{-1 / 2}\right)\right]^{1 / 2}
$$

and $\left|\mathbf{q}_{0}, \mathbf{p}_{0}\right\rangle$ and $\left|\mathbf{q}_{t}, \mathbf{p}_{t}\right\rangle$ are coherent states, the wavefunctions for which are given by

$$
\left\langle x \mid \mathbf{q}_{0}, \mathbf{p}_{0}\right\rangle=\left(\frac{2}{\pi}\right)^{3 N / 4}(\operatorname{det} \tilde{\mathbf{A}})^{1 / 4} \exp \left(-\left(\mathbf{x}-\mathbf{q}_{0}\right)^{T} \tilde{\mathbf{A}}\left(\mathbf{x}-\mathbf{q}_{0}\right)+\frac{i}{\hbar} \mathbf{p}_{0}^{T}\left(\mathbf{x}-\mathbf{q}_{0}\right)\right)
$$

Here $\tilde{\mathbf{A}}$ is a (positive definite) width matrix.

Inserting two such Herman-Kluk representations for the propagator into Eq. (2.1) or (2.2) leads to the following double phase space average for the correlation function

$$
\begin{aligned}
C_{A B}(t) & =(2 \pi \hbar)^{-3 N} Z^{-1} \int d \mathbf{p}_{0} \int d \mathbf{q}_{0} \int d \mathbf{p}_{0}^{\prime} \int d \mathbf{q}_{0}^{\prime} C_{t}\left(\mathbf{q}_{0}, \mathbf{p}_{0}\right) C_{t}^{*}\left(\mathbf{q}_{0}^{\prime}, \mathbf{p}_{0}^{\prime}\right)\left\langle\mathbf{q}_{0}, \mathbf{p}_{0}\left|\hat{A}^{\beta}\right| \mathbf{q}_{0}^{\prime}, \mathbf{p}_{0}^{\prime}\right\rangle \\
& \cdot e^{i S_{t}\left(\mathbf{p}_{0}, \mathbf{q}_{0}\right) / \hbar \hbar} e^{-i S_{t}\left(\mathbf{p}_{0}^{\prime}, \mathbf{q}_{0}^{\prime}\right) / \hbar}\left\langle\mathbf{q}_{t}^{\prime}, \mathbf{p}_{t}^{\prime}|\hat{B}| \mathbf{q}_{t}, \mathbf{p}_{t}\right\rangle
\end{aligned}
$$

The phase cancellation here is as severe in this integrand as it is in Eq. (2.6). Shao and Makri ${ }^{4,5}$ introduced an approximate way to evaluate to it by assuming that the dominant contribution to the double phase space average comes from two trajectories, one starting from $\left(\mathbf{q}_{0}, \mathbf{p}_{0}\right)$ and another from $\left(\mathbf{q}_{0}^{\prime}, \mathbf{p}_{0}^{\prime}\right)$, that satisfy the following 'jumps' in coordinates and momenta at time $t$

$$
\mathbf{q}_{t}^{\prime}=\mathbf{q}_{t}-\hbar \frac{\partial B\left(\mathbf{q}_{t}, \mathbf{p}_{t}\right)}{\partial \mathbf{p}_{t}} ; \mathbf{p}_{t}^{\prime}=\mathbf{p}_{t}+\hbar \frac{\partial B\left(\mathbf{q}_{t}, \mathbf{p}_{t}\right)}{\partial \mathbf{q}_{t}}
$$

This assumption yields the Forward-Backward Semiclassical Dynamics (FBSD) method for the correlation function,

$$
\begin{aligned}
C_{A B}^{F B S D}(t)= & (2 \pi \hbar)^{-3 N} Z^{-1} \int d \mathbf{q}_{0} \int d \mathbf{p}_{0}\left\{\left(1+\frac{3 N}{2}\right)\left\langle\mathbf{q}_{0}, \mathbf{p}_{0}\left|\hat{A}^{\beta}\right| \mathbf{q}_{0}, \mathbf{p}_{0}\right\rangle-\right. \\
& \left.2\left\langle\mathbf{q}_{0}, \mathbf{p}_{0}\left|\left(\hat{\mathbf{x}}-\mathbf{q}_{0}\right)^{T} \tilde{\mathbf{A}} \hat{A}^{\beta}\left(\hat{\mathbf{x}}-\mathbf{q}_{0}\right)\right| \mathbf{q}_{0}, \mathbf{p}_{0}\right\rangle\right\} B\left(\mathbf{q}_{t}, \mathbf{p}_{t}\right)
\end{aligned}
$$

The essential remaining task here is to evaluate the coherent state matrix elements of operator $\hat{A}^{\beta}$, which is non-trivial because $\hat{A}^{\beta}$ involves the Boltzmann operator with the total Hamiltonian of the complete system. This is analogous to the problem of computing the multidimensional Fourier transform to obtain the Wigner function for operator $\hat{A}^{\beta}$ in the LSC-IVR approach described above. As in the 
LSC-IVR approach, if the Boltzmann operator is treated exactly, i.e., by Feynman path integration, the coherent matrix cannot be easily evaluated, as in necessary to obtain the distribution of initial conditions for the real time trajectories; Appendix A also discusses this in more detail. Just as for the LSC-IVR approach, it is these various local harmonic approximations to the Boltzmann that allows these matrix elements (or multidimensional Fourier transforms) to computed analytically and thus obtain an explicit result to the distribution of initial conditions $\left(\mathbf{q}_{0}, \mathbf{p}_{0}\right)$ for the real time trajectories.

\section{Thermal Gaussian Approximation}

For an N-particle system described in Eq. (2.3), the thermal (imaginary time) propagator (i.e., coordinate representation of the Boltzmann operator) $e^{-\tau \hat{H}}$ is approximated by Frantsuzov and Mandelshtam as a multi-dimensional Gaussian form ${ }^{23,24}$ :

$$
\left\langle\mathbf{x}\left|e^{-\tau \hat{H}}\right| \mathbf{q}_{\mathbf{0}}\right\rangle=\left(\frac{1}{2 \pi}\right)^{3 N / 2} \frac{1}{\mid \operatorname{det}(\mathbf{G}(\tau))^{1 / 2}} \exp \left(-\frac{1}{2}(\mathbf{x}-\mathbf{q}(\tau))^{T} \mathbf{G}^{-1}(\tau)(\mathbf{x}-\mathbf{q}(\tau))+\gamma(\tau)\right)
$$

where $\mathbf{G}(\tau)$ is an imaginary-time dependent $3 N \times 3 N$ real symmetric and positive-definite matrix, $\mathbf{q}(\tau)$ the center of the Gaussian, and $\gamma(\tau)$ a real scalar function. The parameters are governed by the equations of motion:

$$
\begin{aligned}
& \frac{d}{d \tau} \mathbf{G}(\tau)=-\mathbf{G}(\tau)\left\langle\nabla \nabla^{T} V(\mathbf{q}(\tau))\right\rangle \mathbf{G}(\tau)+\hbar^{2} \mathbf{M}^{-1} \\
& \frac{d}{d \tau} \mathbf{q}(\tau)=-\mathbf{G}(\tau)\langle\nabla V(\mathbf{q}(\tau))\rangle \\
& \frac{d}{d \tau} \gamma(\tau)=-\frac{1}{4} \operatorname{Tr}\left(\left\langle\nabla \nabla^{T} V(\mathbf{q}(\tau))\right\rangle \mathbf{G}(\tau)\right)-\langle V(\mathbf{q}(\tau))\rangle
\end{aligned}
$$

with the notation

$$
\langle h(\mathbf{q})\rangle=\left(\frac{1}{\pi}\right)^{3 N / 2} \frac{1}{|\operatorname{det}(\mathbf{G}(\tau))|^{1 / 2}} \int_{-\infty}^{\infty} \mathbf{d x}\left(-(\mathbf{x}-\mathbf{q}(\tau))^{T} \mathbf{G}^{-1}(\tau)(\mathbf{x}-\mathbf{q}(\tau))\right) h(\mathbf{x})
$$

The initial conditions for the imaginary time propagation are

$$
\mathbf{q}\left(\begin{array}{ll}
\tau & 0
\end{array}\right)=\mathbf{q}_{0} ; \mathbf{G}\left(\begin{array}{ll}
\tau & 0
\end{array}\right)=\hbar^{2} \tau \mathbf{M}^{-1} ; \gamma\left(\begin{array}{ll}
\tau & 0
\end{array}\right)=-\tau V\left(\mathbf{q}_{0}\right)
$$

To insure that the element of the Boltzmann operator $\left\langle\mathbf{x}\left|e^{-\tau \hat{H}}\right| \mathbf{x}^{\prime}\right\rangle$ is symmetric, Frantsuzov and Mandelshtam compound the approximation in Eq. (3.1) twice to obtain 


$$
\begin{aligned}
\left\langle\mathbf{x}\left|e^{-\tau \hat{H}}\right| \mathbf{x}^{\prime}\right\rangle & =\int \mathbf{d q}_{0}\left\langle\mathbf{x}\left|e^{-\tau \hat{H} / 2}\right| \mathbf{q}_{0}\right\rangle\left\langle\mathbf{q}_{0}\left|e^{-\tau \hat{H} / 2}\right| \mathbf{x}^{\prime}\right\rangle \\
& =\int \mathbf{d q}_{0}\left(\frac{1}{2 \pi}\right)^{3 N} \frac{\exp \left(2 \gamma\left(\frac{\tau}{2}\right)\right)}{\left|\operatorname{det}\left(\mathbf{G}\left(\frac{\tau}{2}\right)\right)\right|} \cdot \exp \left(-\frac{1}{2}\left(\mathbf{x}-\mathbf{q}\left(\frac{\tau}{2}\right)\right)^{T} \mathbf{G}^{-1}\left(\frac{\tau}{2}\right)\left(\mathbf{x}-\mathbf{q}\left(\frac{\tau}{2}\right)\right)\right) \\
& \cdot \exp \left(-\frac{1}{2}\left(\mathbf{x}^{\prime}-\mathbf{q}\left(\frac{\tau}{2}\right)\right)^{T} \mathbf{G}^{-1}\left(\frac{\tau}{2}\right)\left(\mathbf{x}^{\prime}-\mathbf{q}\left(\frac{\tau}{2}\right)\right)\right)
\end{aligned}
$$

The expression for a partition function, for example, becomes

$$
Z=\int d \mathbf{x} \int d \mathbf{q}_{0}\left\langle\mathbf{x}\left|e^{-\tau \hat{H} / 2}\right| \mathbf{q}_{0}\right\rangle\left\langle\mathbf{q}_{0}\left|e^{-\tau \hat{H} / 2}\right| \mathbf{x}\right\rangle=\int d \mathbf{q}_{0} \frac{1}{(4 \pi)^{3 N / 2}} \frac{\exp \left(2 \gamma\left(\frac{\tau}{2}\right)\right)}{\left|\operatorname{det} \mathbf{G}\left(\frac{\tau}{2}\right)\right|^{1 / 2}}
$$

Frantsuzov and Mandelshtam ${ }^{23}$ originally utilized the variational principle to obtain the equations of motion Eq (3.2). Shao and Pollak later rederived these equations by expanding the potential function in terms of the Gaussian averaged potential and its derivatives. In doing so, they showed the TGA to be a harmonic approximation about the imaginary time dependent path $\mathbf{q}(\tau)$, and gave its more generalized version $^{27}$.

In order to make it feasible to apply the TGA to complex systems, one must be able to evaluate

the quantities $\langle V(\mathbf{q}(\tau))\rangle,\langle\nabla V(\mathbf{q}(\tau))\rangle$ and $\left\langle\nabla \nabla^{T} V(\mathbf{q}(\tau))\right\rangle$ in Eq. (3.2) efficiently. To do so, the potential is usually expressed as a sum of Gaussion functions or polynomial functions so that these quantities are evaluated analytically. Recent applications have shown the TGA to be a good approximation for the thermodynamics properties of some complex systems (neon clusters) even at very low temperature ${ }^{23,34}$.

\section{SC-IVR methods with TGA}

The SC-IVR description of real time dynamics can be combined with any type of method for evaluating elements of Boltzmann operator; what one uses for it is a question of accuracy and ease of application. Here we consider the TGA for the Boltzman operator, showing how it leads to particularly simple ways for carrying out semiclassical dynamics calculations for complex systems with the two approximate SC-IVR's, the LSC-IVR and the FBSD (TGA-LSC-IVR or TGA-FBSD), summarized in the preceding Section.

\section{(a) TGA-LSC-IVR}

The TGA for the Boltzmann operator, Eq. (3.5), makes it possible to analytically integrate out the phase term in the Wigner transform of the Boltzmann operator of the LSC-IVR; i.e., substituting Eq. (3.5) into Eq. (2.9) gives

$$
\begin{aligned}
{\left[e^{-\beta \hat{H}}\right]_{w}(\mathbf{x}, \mathbf{p})=} & (2 \pi \hbar)^{-3 N} \int d \Delta \mathbf{x}\left\langle\mathbf{x}-\Delta \mathbf{x} / 2\left|e^{-\beta \hat{H}}\right| \mathbf{x}+\Delta \mathbf{x} / 2\right\rangle e^{i \mathbf{p}^{T} \ddot{\mathbf{A}} / \hbar} \\
\approx & (2 \pi \hbar)^{-3 N} \int d \Delta \mathbf{x} \int d \mathbf{q}\left\langle\mathbf{x}-\Delta \mathbf{x} / 2\left|e^{-\beta \hat{H} / 2}\right| \mathbf{q}_{0}\right\rangle\left\langle\mathbf{q}_{0}\left|e^{-\beta \hat{H} / 2}\right| \mathbf{x}+\Delta \mathbf{x} / 2\right\rangle e^{i \mathbf{p}^{T} \ddot{A} \mathbf{x} / \hbar} \\
& =\int d \mathbf{q}_{0}(2 \pi \hbar)^{-3 N}\left(\frac{1}{\pi}\right)^{3 N / 2} \frac{\exp \left(2 \gamma\left(\frac{\beta}{2}\right)\right)}{\left.\mid \operatorname{det} \mathbf{G}\left(\frac{\beta}{2}\right)\right)^{1 / 2}} \exp \left(-\mathbf{p}^{T} \mathbf{G}\left(\frac{\beta}{2}\right) \mathbf{p} / \hbar^{2}\right) \\
& \cdot \exp \left(-\left(\mathbf{x}-\mathbf{q}\left(\frac{\beta}{2}\right)\right)^{T} \mathbf{G}^{-1}\left(\frac{\beta}{2}\right)\left(\mathbf{x}-\mathbf{q}\left(\frac{\beta}{2}\right)\right)\right)
\end{aligned}
$$


Eq. (4.1) contains no oscillatory term, so the integrand can be naturally used as the sampling function for Monte Carlo calculations of the LSC-IVR correlation functions of complex systems. In the high temperature limit, $\beta \rightarrow 0$, it is straightforward to verify that Eq. (4.1) reduces to its classical limit, the classical Boltzmann distribution, which was also pointed out by Shao and Pollak ${ }^{27}$ by considering the limit $\hbar \rightarrow 0$.

To obtain the Wigner function for operator $\hat{A}^{\beta}$ [Eq 2.8] with the TGA, it is more convenient to use the symmetrized version $\hat{A}^{\beta}=e^{-\beta \hat{H} / 2} \hat{A} e^{-\beta \hat{H} / 2}$ if $\hat{A}=A(\hat{\mathbf{x}})$ is a local operator; however the form $\hat{A}^{\beta}=e^{-\beta \hat{H}} \hat{A}$ is preferred if $\hat{A}=\hat{\mathbf{p}}$, since evaluating derivatives of $\left\langle\mathbf{q}_{0}\left|e^{-\beta \hat{H} / 2}\right| \mathbf{x}+\Delta \mathbf{x} / 2\right\rangle$ with respect to $\mathbf{q}_{0}$ in Eq (4.1) would require considerably more work in the imaginary time propagation with the TGA, i.e., extra equations of motion for $\partial \mathbf{G}(\tau) / \partial \mathbf{q}_{0}, \partial \mathbf{q}(\tau) / \partial \mathbf{q}_{0}$ etc., would be required in Eq (3.2). Applying the TGA within Eq. (2.9), TGA-LSC-IVR auto-correlation functions are expressed as

$$
\begin{aligned}
C_{A A}^{T G A-L S C-I V R}(t) & =\frac{1}{Z} \int d \mathbf{q}_{0} \frac{1}{(4 \pi)^{3 N / 2}} \frac{\exp \left(2 \gamma\left(\frac{\beta}{2}\right)\right)}{\left|\operatorname{det} \mathbf{G}\left(\frac{\beta}{2}\right)\right|^{1 / 2}} \\
& \cdot \int d \mathbf{x}_{0} \frac{1}{\pi^{3 N / 2} \mid \operatorname{det} \mathbf{G}\left(\frac{\beta}{2}\right)^{1 / 2}} \exp \left(-\left(\mathbf{x}_{0}-\mathbf{q}\left(\frac{\beta}{2}\right)\right)^{T} \mathbf{G}^{-1}\left(\frac{\beta}{2}\right)\left(\mathbf{x}_{0}-\mathbf{q}\left(\frac{\beta}{2}\right)\right)\right) \\
& \cdot \int d \mathbf{p}_{0} \frac{\mid \operatorname{det} \mathbf{G}\left(\frac{\beta}{2}\right)^{1 / 2}}{\left(\pi \hbar^{2}\right)^{3 N / 2}} \exp \left(-\mathbf{p}_{0}^{T} \mathbf{G}\left(\frac{\beta}{2}\right) \mathbf{p}_{0} / \hbar^{2}\right) \\
& \cdot f_{A A}^{T G A-L S C-I V R}\left(\mathbf{x}_{0}, \mathbf{p}_{0}, \mathbf{q}\left(\frac{\beta}{2}\right) ; t\right)
\end{aligned}
$$

where

$$
f_{A A}^{T G A-L S C-I V R}\left(\mathbf{x}_{0}, \mathbf{p}_{0}, \mathbf{q}\left(\frac{\beta}{2}\right) ; t\right)=A\left(\mathbf{q}_{0}\right) A\left(\mathbf{x}_{t}\left(\mathbf{x}_{0}, \mathbf{p}_{0}\right)\right)
$$

for local operators with $\hat{A}^{\beta}=e^{-\beta \hat{H} / 2} A(\hat{\mathbf{x}}) e^{-\beta \hat{H} / 2}$, and

$$
f_{A A}^{T G A-L S C-I V R}\left(\mathbf{x}_{0}, \mathbf{p}_{0}, \mathbf{q}\left(\frac{\beta}{2}\right) ; t\right)=\mathbf{p}_{0}^{T} \mathbf{p}_{t}\left(\mathbf{x}_{0}, \mathbf{p}_{0}\right)-i \hbar\left(\mathbf{x}_{0}-\mathbf{q}\left(\frac{\beta}{2}\right)\right)^{T} \mathbf{G}^{-1}\left(\frac{\beta}{2}\right) \mathbf{p}_{t}\left(\mathbf{x}_{0}, \mathbf{p}_{0}\right)
$$

for the momentum operator $\hat{A}=\hat{\mathbf{p}}$ with $\hat{A}^{\beta}=e^{-\beta \hat{H}} \hat{\mathbf{p}}$.

Monte Carlo (MC) evaluation of Eq. (4.2) for complex systems is now straightforward:

(1) Generate an imaginary time trajectory governed by the TGA equations of motion, Eq. (3.2), the weight of which is sampled by the function $\exp \left(2 \gamma\left(\frac{\beta}{2}\right)\right) / \mid \operatorname{det} \mathbf{G}\left(\frac{\beta}{2}\right)^{1 / 2}$.

(2) The imaginary time trajectory produces Gaussian distributions in both position and momentum space, $\exp \left(-\left(\mathbf{x}_{0}-\mathbf{q}\left(\frac{\beta}{2}\right)\right)^{T} \mathbf{G}^{-1}\left(\frac{\beta}{2}\right)\left(\mathbf{x}_{0}-\mathbf{q}\left(\frac{\beta}{2}\right)\right)\right)$ and $\exp \left(-\mathbf{p}_{0}^{T} \mathbf{G}\left(\frac{\beta}{2}\right) \mathbf{p}_{0} / \hbar^{2}\right)$, respectively, which can be use to sample initial conditions $\left(\mathbf{x}_{0}, \mathbf{p}_{0}\right)$ for the real time trajectory very efficiently. 
(3) Run real time trajectories from phase space points $\left(\mathbf{x}_{0}, \mathbf{p}_{0}\right)$ and estimate the property $f_{A A}^{T G A-L S C-I V R}\left(\mathbf{x}_{0}, \mathbf{p}_{0}, \mathbf{q}\left(\frac{\beta}{2}\right) ; t\right)$ of the corresponding time correlation function.

A schematic representation of Eq. (4.2) for the TGA-LSC-IVR is given in Fig. 1. Provided that $f_{A A}^{T G A-L S C-I V R}\left(\mathbf{x}_{0}, \mathbf{p}_{0}, \mathbf{q}\left(\frac{\beta}{2}\right) ; t\right)$ does not vary rapidly, the MC sampling of Eq. (4.2) is much more efficient for high-dimensional system than one might expect. Our applications of the TGA-LSC-IVR to large systems show that only a few phase space points $\left(\mathbf{x}_{0}, \mathbf{p}_{0}\right)$ (i.e., real time trajectories) are necessary for each imaginary time trajectory to yield converged results so long as the number of imaginary time trajectories is sufficient to guarantee the convergence of the thermodynamic properties. For example, when enough imaginary time trajectories have been propagated for the quantity $\left\langle\mathbf{p}^{2}\right\rangle$ to converge, only a few real time trajectories for each imaginary time trajectory are necessary to obtain the real time correlation function $<\mathbf{p}_{0}^{T} \mathbf{p}_{t}>$ accurately.

It should be noted that Borgis and collaborators ${ }^{35}$ have recently expressed the Wigner function of the Boltzmann operator using an imaginary time Gaussian wavepacket approximation that, though formulated differently, can be shown to be equivalent to the TGA treatment (i.e., Eq. (4.1)), and have used it to compute thermal time correlation functions. The essential difference of this work from the present is that ref. 35 uses the Gaussian approximation to compute the Wigner function of the Boltzmann operator itself, and makes the further approximation that the Wigner function of operator $\hat{A}^{\beta}$ is the product of the separate Wigner functions of the Boltzmann opertor and that of operator $\hat{A}$. The TGALSC-IVR approach described above deals directly with the Wigner function of operator $\hat{A}^{\beta}$.

\section{(b) TGA-FBSD}

The Husimi transform of the Boltzmann operator, $\left\langle\mathbf{x}_{0}, \mathbf{p}_{0}\left|e^{-\beta \hat{H}}\right| \mathbf{x}_{0}, \mathbf{p}_{0}\right\rangle$, plays the analogous role in the FBSD method as the Wigner function of operator $\hat{A}^{\beta}$ does in the LSC-IVR; i.e., it is the quantity used to sample initial conditions $\left(\mathbf{x}_{0}, \mathbf{p}_{0}\right)$ for the real time trajectories. Using the TGA [Eq 3.5] to approximate the Boltzmann operator allows the Husimi transform to be evaluated analytically, giving the following result

$$
\begin{aligned}
\left\langle\mathbf{x}_{0}, \mathbf{p}_{0}\left|e^{-\beta \hat{H}}\right| \mathbf{x}_{0}, \mathbf{p}_{0}\right\rangle= & \int d \mathbf{x} \int d \mathbf{x}^{\prime}\left\langle\mathbf{x}_{0}, \mathbf{p}_{0} \mid \mathbf{x}\right\rangle\left\langle\mathbf{x}\left|e^{-\beta \hat{H}}\right| \mathbf{x}^{\prime}\right\rangle\left\langle\mathbf{x}^{\prime} \mid \mathbf{x}_{0}, \mathbf{p}_{0}\right\rangle \\
& =\int d \mathbf{q}_{0} \int d \mathbf{x} \int d \mathbf{x}^{\prime}\left\langle\mathbf{x}_{0}, \mathbf{p}_{0} \mid \mathbf{x}\right\rangle\left\langle\mathbf{x}\left|e^{-\beta \hat{H} / 2}\right| \mathbf{q}_{0}\right\rangle\left\langle\mathbf{q}_{0}\left|e^{-\beta \hat{H} / 2}\right| \mathbf{x}^{\prime}\right\rangle\left\langle\mathbf{x}^{\prime} \mid \mathbf{x}_{0}, \mathbf{p}_{0}\right\rangle \\
& =\int d \mathbf{q}_{0}\left(\frac{1}{2 \pi}\right)^{3 N / 2} \frac{\exp \left(2 \gamma\left(\frac{\beta}{2}\right)\right)|\operatorname{det} \tilde{\mathbf{A}}|^{1 / 2}}{\left|\operatorname{det} \mathbf{G}\left(\frac{\beta}{2}\right)\right| \mid \operatorname{det}\left(\tilde{\mathbf{A}}+\frac{1}{2} \mathbf{G}^{-1}\left(\frac{\beta}{2}\right)\right)} \\
& \cdot \exp \left(-\mathbf{p}_{0}^{T}\left(\tilde{\mathbf{A}}+\frac{1}{2} \mathbf{G}^{-1}\left(\frac{\beta}{2}\right)\right)^{-1} \mathbf{p}_{0} / 2 \hbar^{2}\right) \\
& \cdot \exp \left(-\left(\mathbf{x}_{0}-\mathbf{q}\left(\frac{\beta}{2}\right)\right)^{T} \mathbf{G}^{-1}\left(\frac{\beta}{2}\right)\left(\tilde{\mathbf{A}}+\frac{1}{2} \mathbf{G}^{-1}\left(\frac{\beta}{2}\right)\right)^{-1} \tilde{\mathbf{A}}\left(\mathbf{x}_{0}-\mathbf{q}\left(\frac{\beta}{2}\right)\right)\right)
\end{aligned}
$$

The integrand in Eq. (4.5) can thus be used as the Monte Carlo sampling function for the real time initial conditions $\left(\mathbf{x}_{0}, \mathbf{p}_{0}\right)$. One can show that Eq. (4.5) reduces to its classical limit- the Boltzmann distribution - in the high temperature limit, $\beta \rightarrow 0$, i.e. when Eq. (3.4) holds. 
Combining the TGA with the FBSD expression for the correlation [Eq. (2.15)] gives the following TGA-FBSD result for correlation functions of complex systems

$$
\begin{aligned}
C_{A A}^{T G A-F B S D}(t) & =\frac{1}{Z} \int d \mathbf{q}_{0} \frac{1}{(4 \pi)^{3 N / 2}} \frac{\exp \left(2 \gamma\left(\frac{\beta}{2}\right)\right)}{\left|\operatorname{det} \mathbf{G}\left(\frac{\beta}{2}\right)\right|^{1 / 2}} \\
& \cdot \int d \mathbf{x}_{0} \frac{|\operatorname{det} \tilde{\mathbf{A}}|^{1 / 2} \exp \left(-\left(\mathbf{x}_{0}-\mathbf{q}\left(\frac{\beta}{2}\right)\right)^{T} \mathbf{G}^{-1}\left(\frac{\beta}{2}\right)\left(\tilde{\mathbf{A}}+\frac{1}{2} \mathbf{G}^{-1}\left(\frac{\beta}{2}\right)\right)^{-1} \tilde{\mathbf{A}}\left(\mathbf{x}_{0}-\mathbf{q}\left(\frac{\beta}{2}\right)\right)\right)}{\pi^{3 N / 2}\left|\operatorname{det} \mathbf{G}\left(\frac{\beta}{2}\right)\right|^{1 / 2} \mid \operatorname{det}\left(\tilde{\mathbf{A}}+\frac{1}{2} \mathbf{G}^{-1}\left(\frac{\beta}{2}\right)\right)^{1 / 2}} \\
& \cdot \int d \mathbf{p}_{0} \frac{\exp \left(-\mathbf{p}_{0}^{T}\left(\tilde{\mathbf{A}}+\frac{1}{2} \mathbf{G}^{-1}\left(\frac{\beta}{2}\right)\right)^{-1} \mathbf{p}_{0} / 2 \hbar^{2}\right)}{\left(2 \pi \hbar^{2}\right)^{3 N / 2} \mid \operatorname{det}\left(\tilde{\mathbf{A}}+\frac{1}{2} \mathbf{G}^{-1}\left(\frac{\beta}{2}\right)\right)^{1 / 2}} \\
& \cdot f_{A A}^{T G A-F B S D}\left(\mathbf{x}_{0}, \mathbf{p}_{0}, \mathbf{q}\left(\frac{\beta}{2}\right) ; t\right)
\end{aligned}
$$

where

$$
\begin{aligned}
& f_{A A}^{T G A-F B S D}\left(\mathbf{x}_{0}, \mathbf{p}_{0}, \mathbf{q}\left(\frac{\beta}{2}\right) ; t\right) \\
= & {\left[1+\frac{3 N}{2}-\frac{1}{2}\left(\mathbf{x}_{0}-\mathbf{q}\left(\frac{\beta}{2}\right)\right)^{T} \mathbf{G}^{-1}\left(\frac{\beta}{2}\right)\left(\tilde{\mathbf{A}}+\frac{1}{2} \mathbf{G}^{-1}\left(\frac{\beta}{2}\right)\right)^{-1} \tilde{\mathbf{A}}\left(\tilde{\mathbf{A}}+\frac{1}{2} \mathbf{G}^{-1}\left(\frac{\beta}{2}\right)\right)^{-1} \mathbf{G}^{-1}\left(\frac{\beta}{2}\right)\left(\mathbf{x}_{0}-\mathbf{q}\left(\frac{\beta}{2}\right)\right)\right.} \\
& \left.-\frac{1}{2 \hbar^{2}} \mathbf{p}_{0}^{T}\left(\tilde{\mathbf{A}}+\frac{1}{2} \mathbf{G}^{-1}\left(\frac{\beta}{2}\right)\right)^{-1} \tilde{\mathbf{A}}\left(\tilde{\mathbf{A}}+\frac{1}{2} \mathbf{G}^{-1}\left(\frac{\beta}{2}\right)\right)^{-1} \mathbf{p}_{0}\right] A\left(\mathbf{q}_{0}\right) A\left(\mathbf{x}_{t}\left(\mathbf{x}_{0}, \mathbf{p}_{0}\right)\right)
\end{aligned}
$$

for local operators with $\hat{A}^{\beta}=e^{-\beta \hat{H} / 2} A(\hat{\mathbf{x}}) e^{-\beta \hat{H} / 2}$, and

$$
\begin{aligned}
& f_{A A}^{T G A-F B S D}\left(\mathbf{x}_{0}, \mathbf{p}_{0}, \mathbf{q}\left(\frac{\beta}{2}\right) ; t\right) \\
= & {\left[\left(1+\frac{3 N}{2}\right)-\frac{1}{2 \hbar^{2}} \mathbf{p}_{0}^{T}\left(\tilde{\mathbf{A}}+\frac{1}{2} \mathbf{G}^{-1}\left(\frac{\beta}{2}\right)\right)^{-1} \tilde{\mathbf{A}}\left(\tilde{\mathbf{A}}+\frac{1}{2} \mathbf{G}^{-1}\left(\frac{\beta}{2}\right)\right)^{-1} \mathbf{p}_{0}\right.} \\
& \left.-\frac{1}{2}\left(\mathbf{x}_{0}-\mathbf{q}\left(\frac{\beta}{2}\right)\right)^{T} \mathbf{G}^{-1}\left(\frac{\beta}{2}\right)\left(\tilde{\mathbf{A}}+\frac{1}{2} \mathbf{G}^{-1}\left(\frac{\beta}{2}\right)\right)^{-1} \tilde{\mathbf{A}}\left(\tilde{\mathbf{A}}+\frac{1}{2} \mathbf{G}^{-1}\left(\frac{\beta}{2}\right)\right)^{-1} \mathbf{G}^{-1}\left(\frac{\beta}{2}\right)\left(\mathbf{x}_{0}-\mathbf{q}\left(\frac{\beta}{2}\right)\right)\right] \\
& \cdot\left(\mathbf{p}_{t}^{T} \frac{1}{2} \mathbf{G}^{-1}\left(\frac{\beta}{2}\right)\left(\tilde{\mathbf{A}}+\frac{1}{2} \mathbf{G}^{-1}\left(\frac{\beta}{2}\right)\right)^{-1} \mathbf{p}_{0}-i \hbar \mathbf{p}_{t}^{T} \mathbf{G}^{-1}\left(\frac{\beta}{2}\right)\left(\tilde{\mathbf{A}}+\frac{1}{2} \mathbf{G}^{-1}\left(\frac{\beta}{2}\right)\right)^{-1} \tilde{\mathbf{A}}\left(\mathbf{x}_{0}-\mathbf{q}\left(\frac{\beta}{2}\right)\right)\right) \\
+ & \mathbf{p}_{t}^{T} \frac{1}{2} \mathbf{G}^{-1}\left(\frac{\beta}{2}\right)\left(\tilde{\mathbf{A}}+\frac{1}{2} \mathbf{G}^{-1}\left(\frac{\beta}{2}\right)\right)^{-1} \tilde{\mathbf{A}}\left(\tilde{\mathbf{A}}+\frac{1}{2} \mathbf{G}^{-1}\left(\frac{\beta}{2}\right)\right)^{-1}\left(\mathbf{p}_{0}-i \hbar \mathbf{G}^{-1}\left(\frac{\beta}{2}\right)\left(\mathbf{x}_{0}-\mathbf{q}\left(\frac{\beta}{2}\right)\right)\right)
\end{aligned}
$$

for the momentum operator $\hat{A}=\hat{\mathbf{p}}$ with $\hat{A}^{\beta}=e^{-\beta \hat{H}} \hat{\mathbf{p}}$. To obtain better convergence, the partition function $Z$ is calculated by using $f_{A A}^{T G A-F B S D}\left(\mathbf{x}_{0}, \mathbf{p}_{0}, \mathbf{q}\left(\frac{\beta}{2}\right) ; t\right)(\hat{A}=1)$ as the estimator for the Monte Carlo sampling.

The Monte Carlo procedure for evaluating the TGA-FBSD is similar to that of the TGA-LSCIVR described in the previous section. However since $f_{A A}^{F B S D}\left(\mathbf{x}_{0}, \mathbf{p}_{0}, \mathbf{q}\left(\frac{\beta}{2}\right) ; t\right)$ typically involves more cancellation than does the analogous quantity in the TGA-LSC-IVR, the number of real time trajectories required for each imaginary trajectory to obtain convergence is considerably larger than for the TGALSC-IVR. 


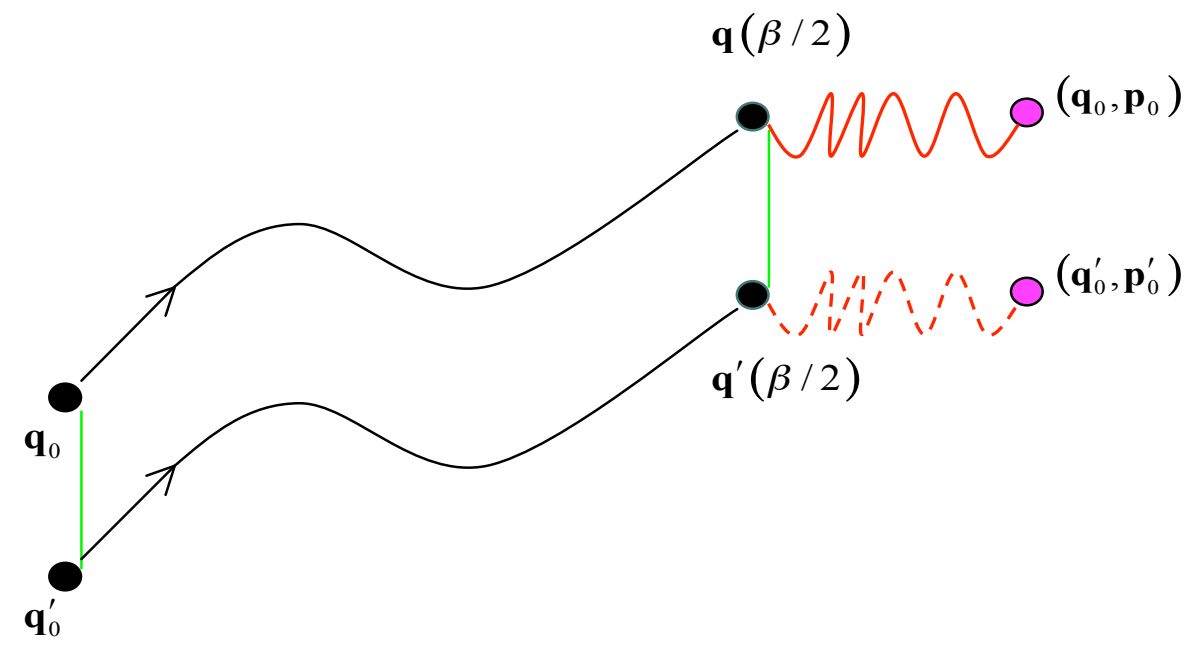

Fig. 1 Schematic representation of the TGA-LSC-IVR representation of the density (Boltzmann operator) for 2 particles. Black solid circles represent positions of 2 particles at the beginning and end of the imaginary time propagation, black curves indicate imaginary time trajectories, purple solid circles demonstrate Wigner density phase points generated upon imaginary time trajectories according to Eq.(4.2), red wavy lines illustrate spring potentials (from Gaussian distributions) between final points of imaginary time trajectories and phase points and green straight lines depict intermolecular interactions. The generated phase points (purple solid circles) are not involved in the intermolecular interaction directly in the imaginary time, which is different from that in Fig. 5.

The schematic representation of the TGA-FBSD representation of the density (Boltzmann operator) for 2 particles is exactly the same as that of the TGA-LSC-IVR, except purple sold circles depict coherent state phase points and the corresponding Gaussian distribution parameters are different.

A schematic representation of Eq. (4.6) for the TGA-FBSD is also given in Fig. 1, which is the same as that of the TGA-LSC-IVR. The TGA-LSC-IVR and the TGA-FBSD are in fact closely related to one another (and give very similar results in most applications). For example, the Gaussian distributions in position and momentum are the same for the two approximations in the limit $\tilde{\mathbf{A}} \rightarrow \infty$ in Eq. (4.6) or (4.5), provided one rescales $\mathbf{p}_{0}$ by the squared root of the matrix $\left(2 \tilde{\mathbf{A}} \mathbf{G}\left(\frac{\beta}{2}\right)+\mathbf{1}\right)^{-1}$. Furthermore, the Wigner function and the Husimi function of the density with the TGA representation are equivalent in the limit $\tilde{\mathbf{A}} \rightarrow \infty$, i.e., the coherent state becomes the position eigenstate, though, $f_{A A}^{T G A-F B S D}\left(\mathbf{x}_{0}, \mathbf{p}_{0}, \mathbf{q}\left(\frac{\beta}{2}\right) ; t\right) \neq f_{A A}^{T G A-L S C-I V R}\left(\mathbf{x}_{0}, \mathbf{p}_{0}, \mathbf{q}\left(\frac{\beta}{2}\right) ; t\right)$ even in that limit. This arises from the fact that the FBSD density is not the same as the Husimi function $\left\langle\mathbf{x}_{0}, \mathbf{p}_{0}\left|\hat{\rho}_{0}\right| \mathbf{x}_{0}, \mathbf{p}_{0}\right\rangle$, but is instead $\left(1+\frac{3 N}{2}\right)\left\langle\mathbf{x}_{0}, \mathbf{p}_{0}\left|\hat{\rho}_{0}\right| \mathbf{x}_{0}, \mathbf{p}_{0}\right\rangle-2\left\langle\mathbf{x}_{0}, \mathbf{p}_{0}\left|\left(\hat{\mathbf{x}}-\mathbf{x}_{0}\right)^{T} \tilde{\mathbf{A}} \hat{\rho}_{0}\left(\hat{\mathbf{x}}-\mathbf{x}_{0}\right)\right| \mathbf{x}_{0}, \mathbf{p}_{0}\right\rangle$, a narrower one. It is straightforward to show that both the TGA-LSC-IVR and the TGA-FBSD reduce to the classical limit at high temperature.

\section{Numerical Applications}

\section{(a) Anharmonic Oscillator}


The first example is a calculation of the force-force autocorrelation function for an asymmetric anharmonic oscillator

$$
V(x)=\frac{1}{2} m \omega^{2} x^{2}-0.10 x^{3}+0.10 x^{4}
$$

with $m=1$ and $\omega=\sqrt{2}$. This potential has been used as a test model and discussed previously in the literature $^{4,16,36}$. Both the LSC-IVR and the FBSD methods are able to describe the dephasing accurately for short times and semiquantitatively for longer times, but fail to capture the rephrasing at longer times due to coherence effects. In many cases in complex systems one expects such long time re-phasing effects to be quenched by coupling among the various degrees of freedom ${ }^{10,20,37}$.

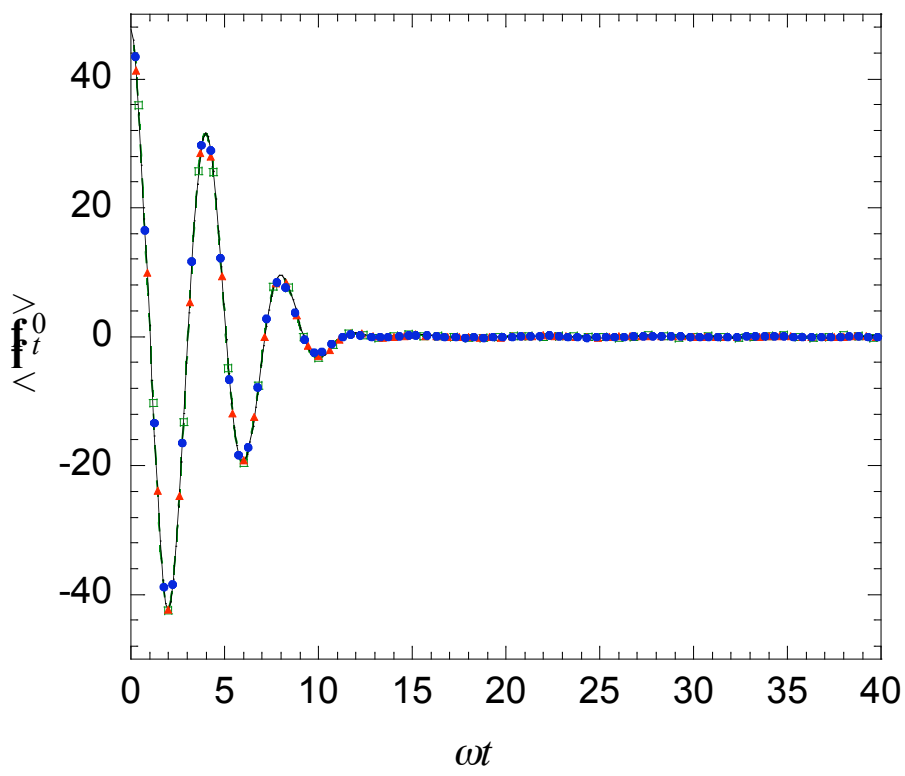

Fig. 2 The symmetrized force autocorrelation function for the one-dimensional anharmonic oscillator given in Eq. (5.1) for $\beta \hbar \omega=\sqrt{2} / 10$. Black line: Exact quantum mechanical result. Green hollow square with dashed line: Classical result. Red solid triangle: TGA-LSC-IVR result. Blue solid circle: TGA-FBSD result.
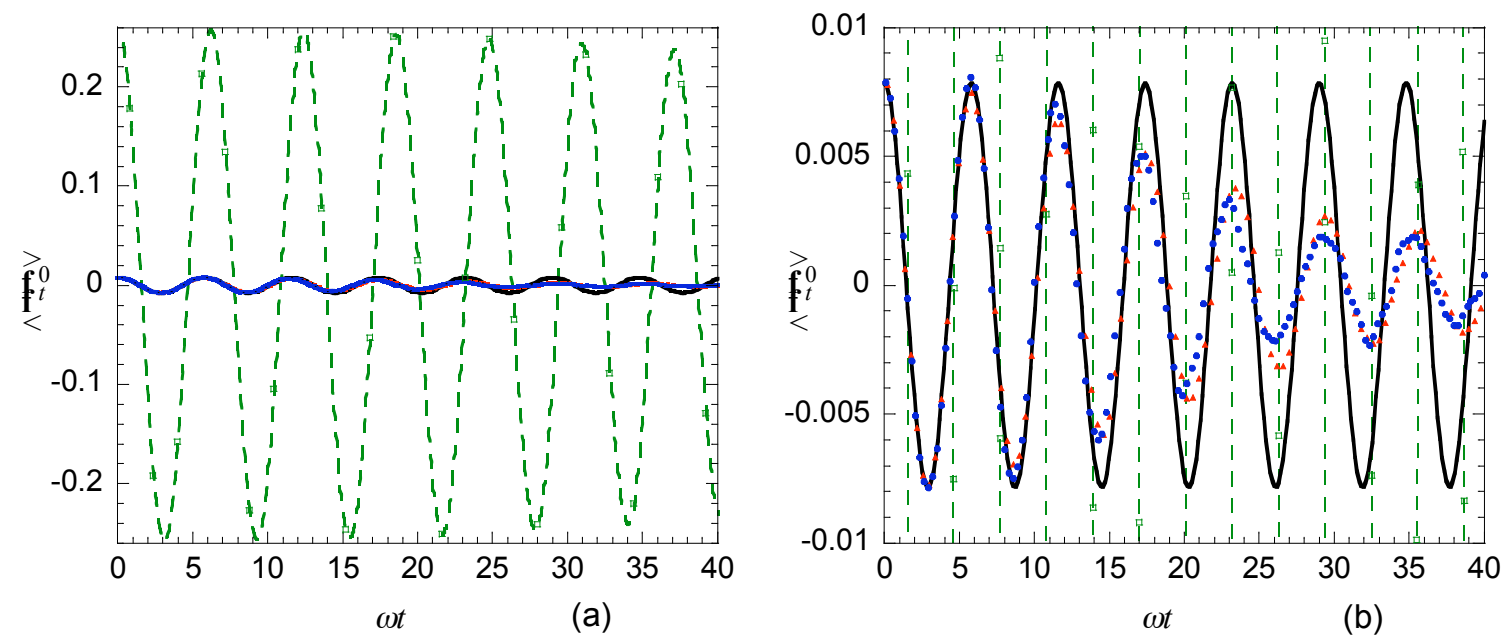
Fig. 3 As in Fig. 2, but for a much lower temperature $\beta \hbar \omega=8 \sqrt{2}$. Panel (b) shows a blowup of the curves shown in (a).

In order to explore how well the TGA-LSC-IVR and the TGA-FBSD perform, we test them at a high temperature $\beta \hbar \omega=\sqrt{2} / 10$ and a very low temperature $\beta \hbar \omega=8 \sqrt{2}$, comparing the results to the classical and the exact quantum results. At both temperatures, we use 21 imaginary trajectories with the imaginary time step of 0.01 , and a large number of real time trajectories generated from each imaginary trajectories with a real time step of 0.02 . The velocity Verlet integrator was used for both real and imaginary time dynamics.

Fig. 2 shows the force autocorrelation function at the temperature $\beta \hbar \omega=\sqrt{2} / 10$. All four results are in good agreement. This is not surprising since both the TGA-LSC-IVR and the TGA-FBSD correlation functions approach the classical result in the high temperature regime where classical mechanics is a good approximation to the exact quantum correlation function. However, at the very low temperature $(\beta \hbar \omega=8 \sqrt{2})$ shown in Fig. 3, the classical results depart from quantum results with regard to both the amplitude of the oscillation (drastically) and frequency (noticeably). It is encouraging to see that the TGA-LSC-IVR and the TGA-FBSD are able to describe these semiquantitatively over several vibrational periods.

\section{(b) Liquid Neon}

Another example is application of the TGA-LSC-IVR and the TGA-FBSD methods to calculate the quantum dynamics with a simulation of liquid neon. Although fully quantum mechanical results on liquids are not available, the FBSD velocity autocorrelation function with the PP approximation (PPFBSD) which accurately describes the zero-time property and satisfies the detailed balance ${ }^{18,38}$ provides a

good comparison. The system is treated as a Lennard-Jones fluid with parameters $\sigma=2.749 \AA$, $\varepsilon / k_{B}=35.6 \mathrm{~K}$ and $m=3.35 \times 10^{-26} \mathrm{~kg}$, at a reduced density $\rho^{*}=0.78$ and temperature $T^{*}=0.84$. This state point is at a fairly low temperature, while still in the liquid region of both the Lennard-Jones and experimental phase diagrams ${ }^{39,40}$. Quantum effects are significant under these conditions: the kinetic energy computed by path integral Monte Carlo methods is about $55.15 \pm 0.27 \mathrm{~K}$, amounting to a $20 \%$ quantum correction to the classical kinetic energy of $44.85 \mathrm{~K}$. These sizable quantum mechanical effects arise from the large zero-point energy of the light neon atoms. The dynamical consequences of these quantum effects are even greater: the momentum correlation function computed by FBSD was found to differ substantially from that obtained by classical molecular dynamics methods, and various quantum correction factor prescriptions give rise to different results, none of which is in good agreement with the FBSD results ${ }^{40}$.

In this application, we used 512,000 imaginary trajectories with 20 imaginary time propagation steps, and one real time trajectory per imaginary time trajectory for the TGA-LSC-IVR (10 real time trajectories per imaginary time trajectory for the TGA-FBSD) with 800 real time propagation steps. During the imaginary time propagation the Lennard-Jones potential is fit by three Gaussian functions the parameters of which are described in the literature ${ }^{23}$. Since liquid neon is treated as an isotropic system, we choose the coherent state parameter $\tilde{\mathbf{A}}=\gamma \mathbf{1}$, where $\mathbf{1}$ is the identity matrix and $\gamma=6$, for both the PPFBSD and the TGA-FBSD simulations. The average kinetic energy computed by the TGA methods is about $54.53 \pm 0.07 \mathrm{~K}$.

Fig. 4 shows the velocity correlation function for liquid neon obtained by the PP-FBSD, the TGA-LSC-IVR and the TGA-FBSD. Both the real and imaginary parts of the correlation function are in good agreement with the PP-FBSD method. The TGA-LSC-IVR correlation function (real part) decays to a somewhat lower well in comparison to the TGA-FBSD and the PP-FBSD results. The comparison of the semiclassical correlation function to the classical result has been shown in Fig. 4. Note that the classical correlation function has no imaginary part, i.e., it is purely real. 
As reported in ref 23, the total number of the imaginary time trajectories in the TGA can be substantially decreased if a classical distribution of $\mathbf{q}_{0}$ is generated at a reference temperature (which can be the same temperature $T=29.90 \mathrm{~K}$ in this case), and in every thousand classical MC steps or so, choose one point $\mathbf{q}_{0}$ for the TGA imaginary time propagation, because the classical Metropolis walk is easy to achieve and thus helps the successive points $\mathbf{q}_{0}$ to sample the space efficiently. As a consequence, it should also greatly accelerate the sampling efficiency in the TGA-LSC-IVR and the TGA-FBSD. We would like to apply this technique in the future.
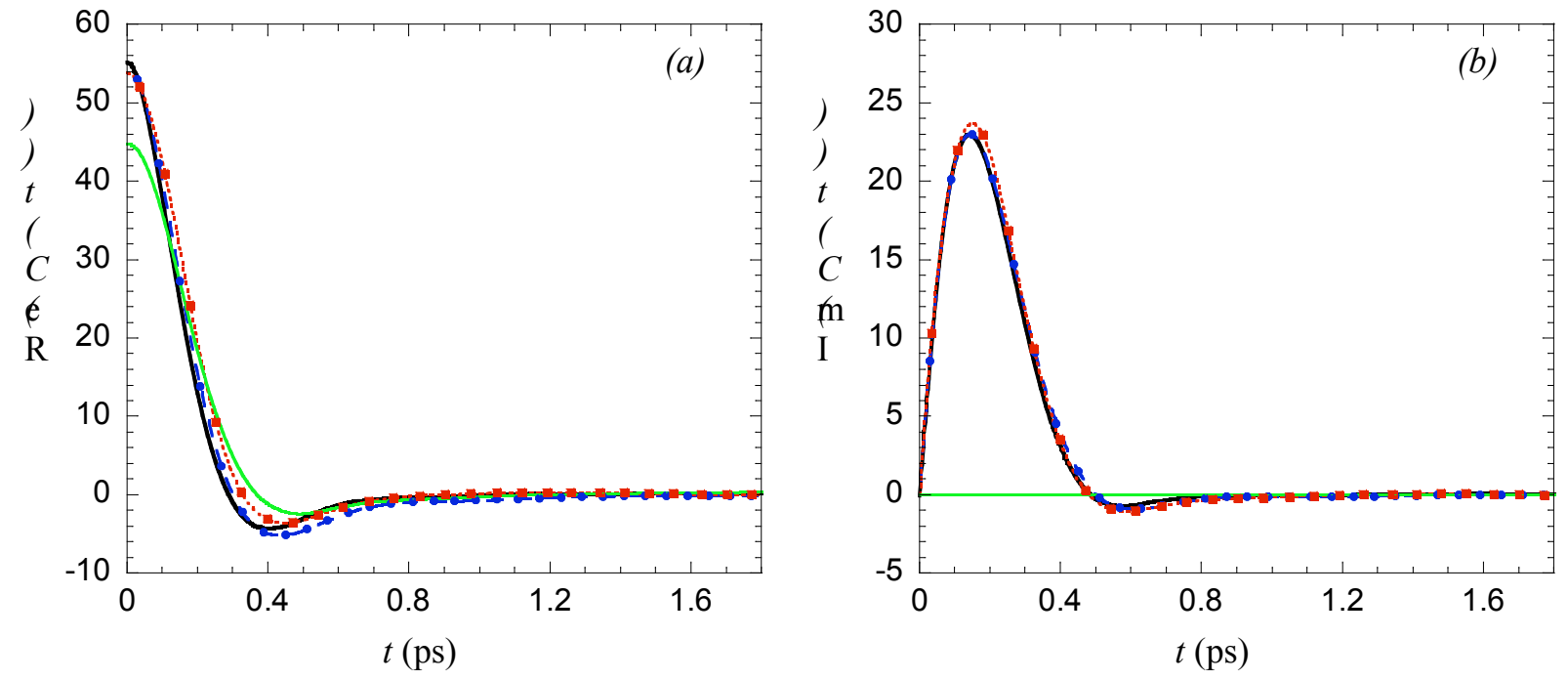

Fig. 4 The velocity autocorrelation function of liquid neon. Panel (a) shows the real part of the correlation function while Panel (b) depicts the imaginary part. Black solid line: PP-FBSD result. Blue solid circle with dashed line: TGA-LSCIVR result. Red solid square with dotted line: TGA-FBSD result. Green solid line: Classical result.

\section{Concluding remarks}

In this paper, we have shown how the TGA can be combined with the SC-IVR to construct time correlation functions in a fully semiclassical scheme, using both real and imaginary time propagations. Specially, we have shown that both the TGA-LSC-IVR and the TGA-FBSD allow one to integrate out the oscillatory term inherent in the LSC-IVR or FBSD, and thus make them practical for descriptions of quantum dynamical effects in large molecular systems. The Kubo-tranformed correlation function can also be calculated in the same fashion without additional work, as shown in Appendix B.

Numerical simulations of an anharmonic oscillator and a low temperature liquid (liquid neon) show that the TGA-LSC-IVR and the TGA-FBSD are good approximations for time correlation functions. Work is continuing to see how well they do in more challenging applications in the condensed matter phase, such as clusters ${ }^{23,34}$ and more quantum mechanical liquids (hydrogen or helium). It will also be interesting in future work to see how application of the TGA can be used with a more rigorous treatment of the real time dynamics in an SC-IVR, such as Miller's version of the forward-backward $I_{V R}^{41}$, or the exact forward-backward semiclassical IVR expression (EFB-IVR) ${ }^{33}$.

We note, however, that the TGA does not provide a good description for the Boltzmann operator in barrier problems, as is typical for the reactive flux correlation functions that determine chemical reaction rates. Current calculations, along with analytical studies (Appendix C), show that the TGA fails to capture the character of the Boltzmann operator at a low temperature: at low temperature, coordinate matrix elements of the Boltzmann operator bifurcate into a dual saddle point structure described by the quantum instanton model. No Gaussian model is able to capture the nature of this bifurcation. The more rigorous imaginary time Van Vleck and the coherent state propagators in imaginary time, however, are 
able to describe this bifurcation semiquantitively ${ }^{42}$. Further effort is thus being devoted to the goal of finding efficient ways of using these more rigorous SC imaginary time approximations for the Boltzmann operator within the overall SC-IVR approach to time correlation functions.

\section{Acknowledgement}

This work was supported by the Office of Naval Research Grant No. N00014-05-1-0457 and by the National Science Foundation Grant No, CHE-0345280. We also acknowledge a generous allocation of supercomputing time from the National Energy Research Scientific Computing Center (NERSC). Thanks are also due to Prof. Eli Pollak for suggesting use of the TGA in SC-IVR correlation functions, and to the US Israel Binational Science Foundation for partial support of the work. 


\section{Appendix A}

As discussed in Section II, the Wigner function for operator $\hat{A}^{\beta}$ is non-trivial since it involves a multi-dimensional Fourier transform involving the Boltzmann operator $e^{-\beta \hat{H}}$ of the complete system. One what to proceed is to express the Boltzmann operator as a Feynman path integral. By inserting path integral beads $\left(\mathbf{q}_{1}, \mathbf{q}_{2}, \cdots, \mathbf{q}_{n}\right)$ for the Boltzmann operator, the path integral representation of the LSCIVR (Eq. (2.8)) may be written as

$$
\begin{gathered}
C_{A B}^{L S C-I V R}(t)=Z^{-1} \int d \mathbf{p}_{0} \int d \mathbf{q}_{0} \int d \mathbf{q}_{1} \cdots \int d \mathbf{q}_{n} \Theta\left(\mathbf{p}_{0}, \mathbf{q}_{0}, \mathbf{q}_{1}, \cdots, \mathbf{q}_{n}\right) \\
\cdot \Lambda_{A B}\left(\mathbf{p}_{0}, \mathbf{q}_{0}, \mathbf{q}_{1}, \cdots, \mathbf{q}_{n}\right)
\end{gathered}
$$

where $\Lambda_{A B}\left(\mathbf{p}_{0}, \mathbf{q}_{0}, \mathbf{q}_{1}, \cdots, \mathbf{q}_{n}\right)$ is function related with the operators $\hat{A}$ and $\hat{B}$, and the sampling function $\Theta\left(\mathbf{p}_{0}, \mathbf{q}_{0}, \mathbf{q}_{1}, \cdots, \mathbf{q}_{n}\right)$ is the Fourier transform of the integrand of $\left\langle\mathbf{q}_{0}-\ddot{\mathbf{A}} \mathbf{q}_{0} / 2\left|e^{-\beta \hat{H}}\right| \mathbf{q}_{0}+\ddot{\mathbf{A}} \mathbf{q}_{0} / 2\right\rangle$ after inserting the beads $\left(\mathbf{q}_{1}, \mathbf{q}_{2}, \cdots, \mathbf{q}_{n}\right)$ into the elements of the Boltzmann operator, which gives

$$
\begin{aligned}
\Theta\left(\mathbf{p}_{0}, \mathbf{q}_{0}, \mathbf{q}_{1}, \cdots, \mathbf{q}_{n}\right)= & (2 \pi \hbar)^{-3 N} \int d \ddot{\mathbf{A}} \mathbf{q}_{0} e^{i \mathbf{p}_{0}^{\mathrm{T}} \ddot{\mathbf{q}_{0}}}\left\langle\mathbf{q}_{0}-\ddot{\mathbf{A}} \mathbf{q}_{0} / 2\left|e^{-\Delta \beta \hat{H}}\right| \mathbf{q}_{1}\right\rangle\left\langle\mathbf{q}_{1}\left|e^{-\Delta \beta \hat{H}}\right| \mathbf{q}_{2}\right\rangle \\
& \cdots\left\langle\mathbf{q}_{n}\left|e^{-\Delta \beta \hat{H}}\right| \mathbf{q}_{0}+\ddot{\mathbf{A}} \mathbf{q}_{0} / 2\right\rangle
\end{aligned}
$$

where $\Delta \beta=\beta /(n+1)$. Fig. 5 shows a schematic representation of Eq. (A.2).

For complex systems, generally it is not possible to explicitly evaluate the Fourier transform in Eq. (2.9) or Eq. (A.2) because of the sign problem (the phase cancellation is severe). Some kinds of harmonic approximation $^{3,6,7}$ for the elements of the Boltzmann operator is necessary. These approximations have been successfully applied to some complex systems ${ }^{15,21}$. They all, however, encounter problems at low temperature when the potential energy has negative curvature; this shows up most strikingly in regions of potential barriers, but also in the long range region of bounded potentials. 


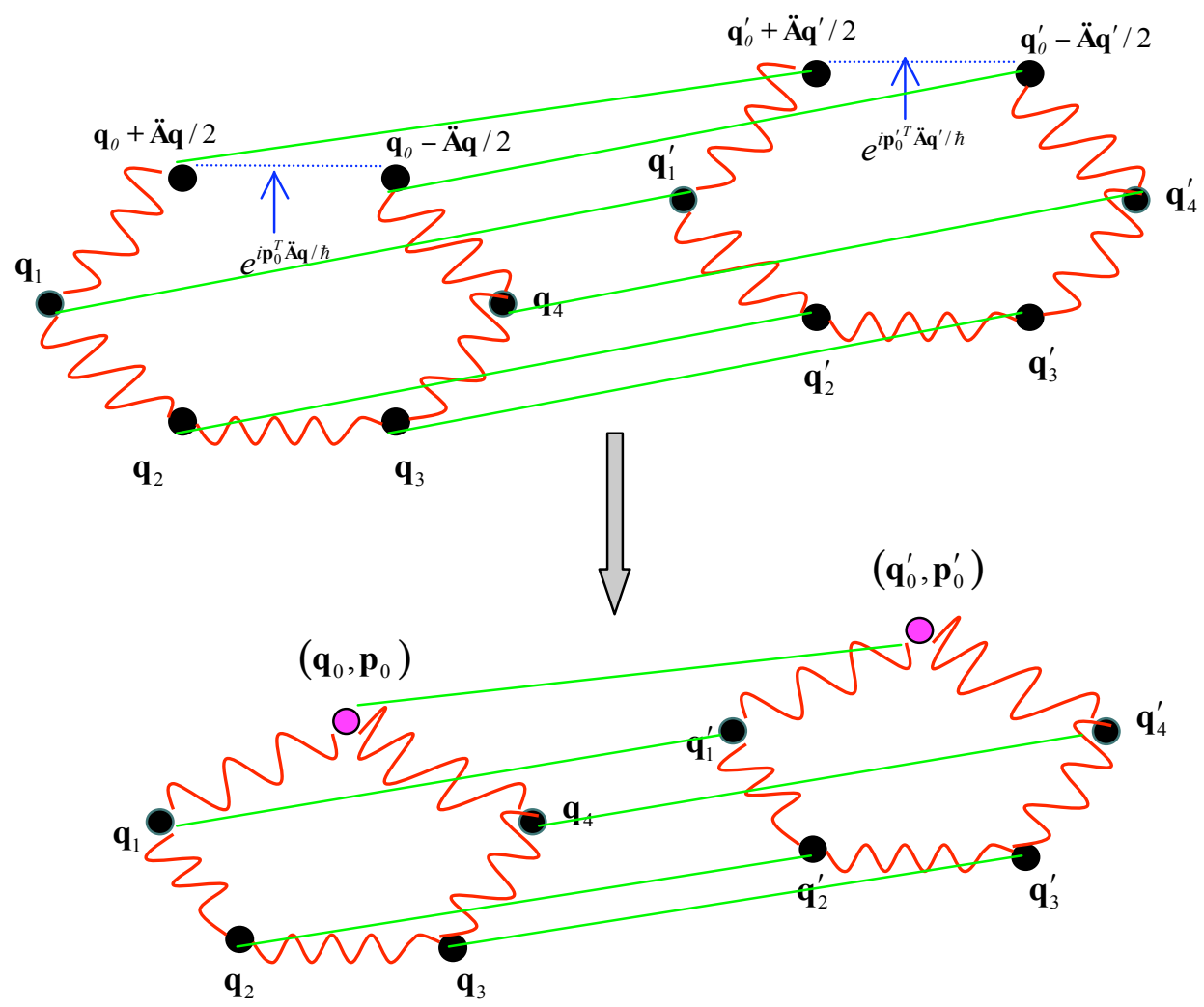

Fig. 5 Schematic representation of combined path integral LSC-IVR representation of the density (Boltzmann operator) for 2 particles for $n=4$ inserting beads. Black solid circles represent the path integral beads, red wavy lines indicate spring potentials between neighbour beads, green straight lines depict intermolecular interactions and blue dotted lines demonstrate the connection between the end beads via the Fourier transform. The open rings illustrate the integrand in Eq. (A.2), while the close ones the Wigner function of the Boltzmann operator. Note the two end beads of the open rings merge into single beads (purple solid circles) at their average positions associated with the momenta coming from the Fourier transform of the difference between the two end beads, thus in this way it constructs the initial phase space in the LSC-IVR.

A similar difficulty arises in the FBSD if one uses a Feynman path integral representation of the Boltzmann operator. Introducing the path integral representation of the Boltzmann operator into Eq. (2.15) yields the following form ${ }^{44}$ for the correlation function

$$
\begin{aligned}
& C_{A B}^{F B S D}(t)=(2 \pi \hbar)^{-3 N} Z^{-1} \int d \mathbf{p}_{0} \int d \mathbf{q}_{0} \int d \mathbf{q}_{1} \cdots \int d \mathbf{q}_{n} \Theta\left(\mathbf{p}_{0}, \mathbf{q}_{0}, \mathbf{q}_{1}, \cdots, \mathbf{q}_{n}\right) \\
& \cdot \Lambda_{A B}\left(\mathbf{p}_{0}, \mathbf{q}_{0}, \mathbf{q}_{1}, \cdots, \mathbf{q}_{n}\right)
\end{aligned}
$$

where $\Lambda_{A B}\left(\mathbf{p}_{0}, \mathbf{q}_{0}, \mathbf{q}_{1}, \cdots, \mathbf{q}_{n}\right)$ is a function related to the operators $\hat{A}$ and $\hat{B}$, and $\Theta\left(\mathbf{p}_{0}, \mathbf{q}_{0}, \mathbf{q}_{1}, \cdots, \mathbf{q}_{n}\right)$ is the integrand of $\left\langle\mathbf{q}_{0}, \mathbf{p}_{0}\left|e^{-\beta \hat{H}}\right| \mathbf{q}_{0}, \mathbf{p}_{0}\right\rangle$ after inserting the beads $\left(\mathbf{q}_{1}, \mathbf{q}_{2}, \cdots, \mathbf{q}_{n}\right)$ into the Boltzmann operator, which has the explicit form 


$$
\begin{aligned}
\Theta\left(\mathbf{p}_{0}, \mathbf{q}_{0}, \mathbf{q}_{1}, \cdots, \mathbf{q}_{n}\right)= & \left\langle\mathbf{q}_{0}, \mathbf{p}_{0}\left|e^{-\Delta \beta \hat{H_{0} / 2}}\right| \mathbf{q}_{1}\right\rangle e^{-\Delta \beta V\left(\mathbf{q}_{1}\right)}\left\langle\mathbf{q}_{1}\left|e^{-\Delta \beta \hat{H_{0} / 2}}\right| \mathbf{q}_{2}\right\rangle \cdots \\
& \cdot e^{-\Delta \beta V\left(\mathbf{q}_{n}\right)}\left\langle x_{n}\left|e^{-\Delta \beta H_{0} / 2}\right| \mathbf{q}_{0}, \mathbf{p}_{0}\right\rangle \\
& \propto \exp \left\{-\left(\mathbf{q}_{1}-\mathbf{q}_{0}\right)^{T} \mathbf{M}\left(\mathbf{M}+\hbar^{2} \Delta \beta \tilde{\mathbf{A}}\right)^{-1} \tilde{\mathbf{A}}\left(\mathbf{q}_{1}-\mathbf{q}_{0}\right)\right. \\
& +\left(\mathbf{q}_{n}-\mathbf{q}_{0}\right)^{T} \mathbf{M}\left(\mathbf{M}+\hbar^{2} \Delta \beta \tilde{\mathbf{A}}\right)^{-1} \tilde{\mathbf{A}}\left(\mathbf{q}_{n}-\mathbf{q}_{0}\right) \\
& +\frac{\Delta \beta}{2} \mathbf{p}_{0}^{T}\left(\mathbf{M}+\hbar^{2} \Delta \beta \tilde{\mathbf{A}}\right)^{-1} \mathbf{p}_{0}+\frac{i}{\hbar} \mathbf{p}_{0}^{T}\left(\mathbf{M}+\hbar^{2} \Delta \beta \tilde{\mathbf{A}}\right)^{-1} \mathbf{M}\left(\mathbf{q}_{1}-\mathbf{q}_{n}\right) \\
& \left.-\frac{1}{2 \hbar^{2} \Delta \beta} \sum_{k=2}^{n}\left(\mathbf{q}_{k}-\mathbf{q}_{k-1}\right)^{T} \mathbf{M}\left(\mathbf{q}_{k}-\mathbf{q}_{k-1}\right)-\Delta \beta \sum_{k=1}^{n} V\left(\mathbf{q}_{k}\right)\right\}
\end{aligned}
$$

Usually $\Theta\left(\mathbf{p}_{0}, \mathbf{q}_{0}, \mathbf{q}_{1}, \cdots, \mathbf{q}_{n}\right) \mid$ would be used as the sampling function for the Monte Carlo evaluation of the integral in Eq. (A.3). However, the phase in Eq. (A.4) only vanishes when inserting one bead is sufficient for evaluating the Boltzmann operator, and thus becomes a bottleneck at low temperature for complex systems. Nakayama and Makri therefore introduced the pair-product (PP) approximation so that one bead is accurate enough for low-temperature pure isotropic liquids ${ }^{17}$. Fig. 6 gives a schematic representation of Eq. (A.4).

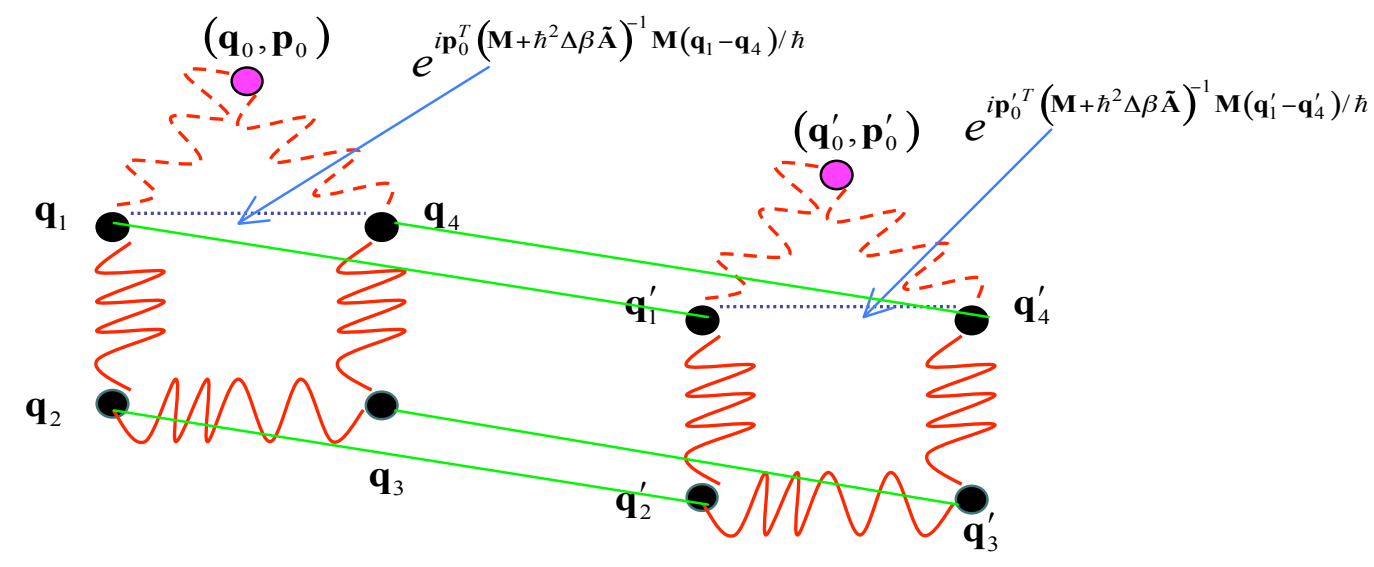

Fig. 6 Schematic representation of combined path integral FBSD representation of the density (Boltzmann operator) for 2 particles for $n=4$ inserting beads based on an early version ${ }^{16}$. Black solid circles represent the path integral beads, purple solid circles demonstrate the coherent state beads, red solid wavy lines indicate spring potentials between neighbor path integral beads while red dashed wavy lines illustrate the spring potential between the coherent state bead and its neighbor path integral beads, green straight lines depict intermolecular interactions and blue dotted lines shows the interaction between the two path integral beads that are neighbors to the coherent state bead from the phase term in Eq. (A.4). The coherent state beads are not involved in the intermolecular interaction directly in the imaginary time. Note the coherent state beads define the initial phase space in the FBSD.

To summarize, for general complex systems, the rigorous path integral treatment of the Boltzmann operator in both the LSC-IVR and the FBSD methods encounters the problem - the inability to explicitly obtain the initial phase distribution for the real time trajectories, as shown in Fig. 5 and Fig. 6. 


\section{Appendix B}

The Kubo-transformed real-time correlation function ${ }^{45}$ is given as

$$
C_{A B}^{K u b o}(t)=\frac{1}{Z} \operatorname{Tr}\left(\hat{A}_{K u b o}^{\beta} e^{i \hat{H} t / \hbar} \hat{B} e^{-i \hat{H} t / \hbar}\right)
$$

where $\hat{A}_{K u b o}^{\beta}=\frac{1}{\beta} \int_{0}^{\beta} \mathrm{d} \lambda e^{-(\beta-\lambda) \hat{H}} \hat{A} e^{-\lambda \hat{H}}$ is the Kubo-transformed operator. The Kubo-transformed versions of the TGA-LSC-IVR and TGA-FBSD can both be expressed in a similar form as Eq. (4.2) or Eq. (4.6). Here we take the momentum and the force auto correlation functions as examples. Since the momentum and force operators can be expressed as $\hat{\mathbf{p}}=\frac{i}{\hbar} \mathbf{M}[\hat{H}, \hat{\mathbf{x}}]$ and $\mathbf{F}(\hat{\mathbf{x}})=-V^{\prime}(\hat{\mathbf{x}})=\frac{i}{\hbar}[\hat{H}, \hat{\mathbf{p}}]$, their Kubo transforms are given by

$$
\hat{\mathbf{p}}_{\text {Kubo }}^{\beta}=\frac{i}{\hbar \beta} \mathbf{M}\left[\hat{\mathbf{x}}, e^{-\beta \hat{H}}\right]
$$

and

$$
\hat{\mathbf{F}}_{\text {Kubo }}^{\beta}=\frac{i}{\hbar \beta}\left[\hat{\mathbf{p}}, e^{-\beta \hat{H}}\right]
$$

Eq. (B.2) and (B.3) directly connect the standard real-time correlation function (Eq. (2.1)) with the Kubotransformed correlation function (Eq. (B.1)), which shows another way to calculate the Kubo-transformed correlation function.

The Wigner function and the Husimi function of $\hat{\mathbf{p}}_{\text {Kubo }}^{\beta}$ or $\hat{\mathbf{F}}_{\text {Kubo }}^{\beta}$ can be obtained analytically using the TGA in the same way as in Eq. (4.1) and Eq. (4.5) respectively. The final form of the TGA-LSC-IVR of the Kubo-transformed real time correlation function can be shown to be

$$
\begin{aligned}
C_{A A, K u b o}^{T G A-I S C R}(t)= & \frac{1}{Z} \int d \mathbf{q}_{0} \frac{1}{(4 \pi)^{3 N / 2}} \frac{\exp \left(2 \gamma\left(\frac{\beta}{2}\right)\right)}{\left|\operatorname{det} \mathbf{G}\left(\frac{\beta}{2}\right)\right|^{1 / 2}} \\
& \cdot \int d \mathbf{x}_{0} \frac{1}{\pi^{3 N / 2} \mid \operatorname{det} \mathbf{G}\left(\frac{\beta}{2}\right)^{1 / 2}} \exp \left(-\left(\mathbf{x}_{0}-\mathbf{q}\left(\frac{\beta}{2}\right)\right)^{T} \mathbf{G}^{-1}\left(\frac{\beta}{2}\right)\left(\mathbf{x}_{0}-\mathbf{q}\left(\frac{\beta}{2}\right)\right)\right) \\
& \cdot \int d \mathbf{p}_{0} \frac{\mid \operatorname{det} \mathbf{G}\left(\frac{\beta}{2}\right)^{1 / 2}}{\left(\pi \hbar^{2}\right)^{3 N / 2}} \exp \left(-\mathbf{p}_{0}^{T} \mathbf{G}\left(\frac{\beta}{2}\right) \mathbf{p}_{0} / \hbar^{2}\right) \\
& \cdot f_{A A, K u b o}^{T G A-I V C}\left(\mathbf{x}_{0}, \mathbf{p}_{0}, \mathbf{q}\left(\frac{\beta}{2}\right) ; t\right)
\end{aligned}
$$

where

$$
f_{A A, K u b o}^{T G A-L S C-I V R}\left(\mathbf{x}_{0}, \mathbf{p}_{0}, \mathbf{q}\left(\frac{\beta}{2}\right) ; t\right)=\frac{2}{\hbar^{2} \beta} \mathbf{p}_{0}^{T} \mathbf{M G}\left(\frac{\beta}{2}\right) \mathbf{p}_{t}
$$

for the momentum $\hat{A}=\hat{\mathbf{p}}$, and

$$
f_{A A, K u b o}^{T G A-L S C-I V R}\left(\mathbf{x}_{0}, \mathbf{p}_{0}, \mathbf{q}\left(\frac{\beta}{2}\right) ; t\right)=-\frac{2}{\beta}\left(\mathbf{x}_{0}-\mathbf{q}\left(\frac{\beta}{2}\right)\right)^{T} \mathbf{G}\left(\frac{\beta}{2}\right) \mathbf{F}_{t}\left(\mathbf{x}_{0}, \mathbf{p}_{0}\right)
$$


for the force $\hat{A}=\hat{\mathbf{F}}$.

The formula of $C_{A A, K u b o}^{T G A-I V R}(t)$ and that of $C_{A A}^{T G A-L S C-I V R}(t)$ in Eq. (4.2) show that they share exactly the same MC sampling, except that the estimator function $f_{A A, K L L b O}^{T G A-L C L R}\left(\mathbf{x}_{0}, \mathbf{p}_{0}, \mathbf{q}\left(\frac{\beta}{2}\right) ; t\right)$ is different from $f_{A A}^{T G A-L S C-I V R}\left(\mathbf{x}_{0}, \mathbf{p}_{0}, \mathbf{q}\left(\frac{\beta}{2}\right) ; t\right)$, so they are able to be calculated simultaneously.

Similarly, we obtain the following result for the TGA-FBSD version of the Kubo-transformed correlation function

$$
\begin{aligned}
& C_{A A, K u b o}^{T G A-F B S D}(t)=\frac{1}{Z} \int d \mathbf{q}_{0} \frac{1}{(4 \pi)^{3 N / 2}} \frac{\exp \left(2 \gamma\left(\frac{\beta}{2}\right)\right)}{\mid \operatorname{det} \mathbf{G}\left(\frac{\beta}{2}\right)^{1 / 2}} \\
& \int d \mathbf{x}_{0} \frac{|\operatorname{det} \tilde{\mathbf{A}}|^{1 / 2} \exp \left(-\left(\mathbf{x}_{0}-\mathbf{q}\left(\frac{\beta}{2}\right)\right)^{T} \mathbf{G}^{-1}\left(\frac{\beta}{2}\right)\left(\tilde{\mathbf{A}}+\frac{1}{2} \mathbf{G}^{-1}\left(\frac{\beta}{2}\right)\right)^{-1} \tilde{\mathbf{A}}\left(\mathbf{x}_{0}-\mathbf{q}\left(\frac{\beta}{2}\right)\right)\right)}{\left.\pi^{3 N / 2}\left|\operatorname{det} \mathbf{G}\left(\frac{\beta}{2}\right)^{1 / 2}\right| \operatorname{det}\left(\tilde{\mathbf{A}}+\frac{1}{2} \mathbf{G}^{-1}\left(\frac{\beta}{2}\right)\right)\right|^{1 / 2}} \\
& \int d \mathbf{p}_{0} \frac{\exp \left(-\mathbf{p}_{0}^{T}\left(\tilde{\mathbf{A}}+\frac{1}{2} \mathbf{G}^{-1}\left(\frac{\beta}{2}\right)\right)^{-1} \mathbf{p}_{0} / 2 \hbar^{2}\right)}{\left(2 \pi \hbar^{2}\right)^{3 / 2} \mid \operatorname{det}\left(\tilde{\mathbf{A}}+\frac{1}{2} \mathbf{G}^{-1}\left(\frac{\beta}{2}\right)\right)^{1 / 2}} \\
& \cdot f_{A A, K u b o}^{T G A-F B D D}\left(\mathbf{x}_{0}, \mathbf{p}_{0}, \mathbf{q}\left(\frac{\beta}{2}\right) ; t\right)
\end{aligned}
$$

where

$$
\begin{aligned}
& f_{A A, K u b o}^{T G A-F S D}\left(\mathbf{x}_{0}, \mathbf{p}_{0}, \mathbf{q}\left(\frac{\beta}{2}\right) ; t\right) \\
& =\left[\left(1+\frac{3 N}{2}\right)-\frac{1}{2 \hbar^{2}} \mathbf{p}_{0}^{T}\left(\tilde{\mathbf{A}}+\frac{1}{2} \mathbf{G}^{-1}\left(\frac{\beta}{2}\right)\right)^{-1} \tilde{\mathbf{A}}\left(\tilde{\mathbf{A}}+\frac{1}{2} \mathbf{G}^{-1}\left(\frac{\beta}{2}\right)\right)^{-1} \mathbf{p}_{0}\right. \\
& \left.-\frac{1}{2}\left(\mathbf{x}_{0}-\mathbf{q}\left(\frac{\beta}{2}\right)\right)^{T} \mathbf{G}^{-1}\left(\frac{\beta}{2}\right)\left(\tilde{\mathbf{A}}+\frac{1}{2} \mathbf{G}^{-1}\left(\frac{\beta}{2}\right)\right)^{-1} \tilde{\mathbf{A}}\left(\tilde{\mathbf{A}}+\frac{1}{2} \mathbf{G}^{-1}\left(\frac{\beta}{2}\right)\right)^{-1} \mathbf{G}^{-1}\left(\frac{\beta}{2}\right)\left(\mathbf{x}_{0}-\mathbf{q}\left(\frac{\beta}{2}\right)\right)\right] \\
& \cdot\left(\frac{1}{\hbar^{2} \beta} \mathbf{p}_{t}^{T} \mathbf{M}\left(\tilde{\mathbf{A}}+\frac{1}{2} \mathbf{G}^{-1}\left(\frac{\beta}{2}\right)\right)^{-1} \mathbf{p}_{0}\right)+\frac{1}{\hbar^{2} \beta} \mathbf{p}_{t}^{T} \mathbf{M}\left(\tilde{\mathbf{A}}+\frac{1}{2} \mathbf{G}^{-1}\left(\frac{\beta}{2}\right)\right)^{-1} \tilde{\mathbf{A}}\left(\tilde{\mathbf{A}}+\frac{1}{2} \mathbf{G}^{-1}\left(\frac{\beta}{2}\right)\right)^{-1} \mathbf{p}_{0}
\end{aligned}
$$

for the momentum $\hat{A}=\hat{\mathbf{p}}$, and

$$
\begin{aligned}
& f_{A A, K u b o}^{T G A-F S D}\left(\mathbf{x}_{0}, \mathbf{p}_{0}, \mathbf{q}\left(\frac{\beta}{2}\right) ; t\right) \\
& =\left[\left(1+\frac{3 N}{2}\right)-\frac{1}{2 \hbar^{2}} \mathbf{p}_{0}^{T}\left(\tilde{\mathbf{A}}+\frac{1}{2} \mathbf{G}^{-1}\left(\frac{\beta}{2}\right)\right)^{-1} \tilde{\mathbf{A}}\left(\tilde{\mathbf{A}}+\frac{1}{2} \mathbf{G}^{-1}\left(\frac{\beta}{2}\right)\right)^{-1} \mathbf{p}_{0}\right. \\
& \left.-\frac{1}{2}\left(\mathbf{x}_{0}-\mathbf{q}\left(\frac{\beta}{2}\right)\right)^{T} \mathbf{G}^{-1}\left(\frac{\beta}{2}\right)\left(\tilde{\mathbf{A}}+\frac{1}{2} \mathbf{G}^{-1}\left(\frac{\beta}{2}\right)\right)^{-1} \tilde{\mathbf{A}}\left(\tilde{\mathbf{A}}+\frac{1}{2} \mathbf{G}^{-1}\left(\frac{\beta}{2}\right)\right)^{-1} \mathbf{G}^{-1}\left(\frac{\beta}{2}\right)\left(\mathbf{x}_{0}-\mathbf{q}\left(\frac{\beta}{2}\right)\right)\right] \\
& \cdot\left(-\frac{2}{\beta}\left(\mathbf{x}_{0}-\mathbf{q}\left(\frac{\beta}{2}\right)\right)^{T} \tilde{\mathbf{A}}\left(\tilde{\mathbf{A}}+\frac{1}{2} \mathbf{G}^{-1}\left(\frac{\beta}{2}\right)\right)^{-1} \mathbf{G}^{-1}\left(\frac{\beta}{2}\right) \mathbf{F}_{t}\right) \\
& -\frac{1}{\beta}\left(\mathbf{x}_{0}-\mathbf{q}\left(\frac{\beta}{2}\right)\right)^{T} \mathbf{G}^{-1}\left(\frac{\beta}{2}\right)\left(\tilde{\mathbf{A}}+\frac{1}{2} \mathbf{G}^{-1}\left(\frac{\beta}{2}\right)\right)^{-1} \tilde{\mathbf{A}}\left(\tilde{\mathbf{A}}+\frac{1}{2} \mathbf{G}^{-1}\left(\frac{\beta}{2}\right)\right)^{-1} \mathbf{G}^{-1}\left(\frac{\beta}{2}\right) \mathbf{F}_{t}
\end{aligned}
$$

for the force $\hat{A}=\hat{\mathbf{F}}$. 


\section{Appendix C}

It can be shown that the matrix $\mathbf{G}(\tau)$ in Eq. (3.2) is always positive definite for a physical system (for which the second derivative of the potential is zero or positive in the asymptotic region). Therefore, since matrix elements of the Boltzmann operator are given within the TGA by

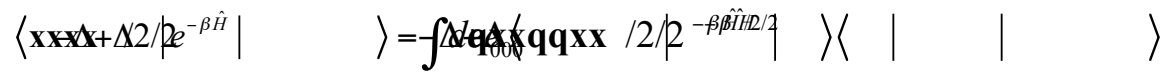

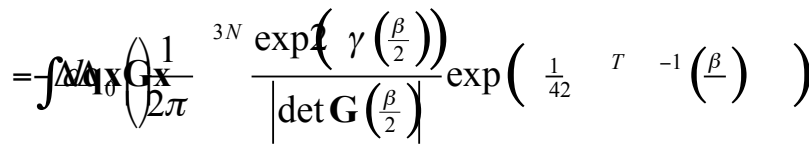

$$
\begin{aligned}
& \cdot \exp \left(\left(\mathbf{x q G x}\left(\boldsymbol{q}_{22}^{\beta \beta}\right)\right)^{T}{ }^{-1}(-)(\quad(-))\right)
\end{aligned}
$$

and since $\mathbf{G}\left(\frac{\beta}{2}\right)$ is positive definite, the matrix element along the off-diagonal direction $\ddot{\mathbf{A}} \mathbf{x}$ is always a Gaussian centered at 0 . This is qualitatively wrong, however, for a barrier potential (i.e. Eckart barrier) at low temperature, where the element $\left\langle-\ddot{\mathbf{A}} \mathbf{x} / 2\left|e^{-\beta \hat{H}}\right| \ddot{\mathbf{A}} \mathbf{x} / 2\right\rangle$ is typically bimodal; i.e., the TGA is intrinsically incapable of capturing the two saddle points (along the off-diagonal direction $\ddot{A} \mathbf{x}$ ) of the coordinate Boltzmann matrix element for a barrier potential, which is characteristic of the low temperature regime, as shown in Fig. 1(a) in ref 46. This bimodal character, however, is described semiquantitively by the more rigorous imaginary time Van Vleck and the Herman-Kluk propagators in imaginary times ${ }^{42}$. 


\section{References}

1 J. Cao and G. A. Voth, J. Chem. Phys. 100, 5106 (1994); S. Jang and G. A. Voth, J. Chem. Phys. 111, 2371 (1999); I. R. Craig and D. E. Manolopoulos, Journal of Chemical Physics 121 (8), 3368 (2004).

2 X. Sun, H. B. Wang, and W. H. Miller, Journal of Chemical Physics 109 (11), 4190 (1998). H. B. Wang, X. Sun, and W. H. Miller, Journal of Chemical Physics 108 (23), 9726 (1998). J. Shao and N. Makri, J. Phys. Chem. A 103, 7753 (1999). J. Shao and N. Makri, J. Phys. Chem. A 103, 9479 (1999). J. A. Poulsen, G. Nyman, and P. J. Rossky, J. Chem. Phys. 119, 12179 (2003). Q. Shi and E. Geva, J. Phys. Chem. A 107, 9059 (2003). J. H. Van Vleck, Proc. Nat. Acad. U.S. Sci. 14, 178 (1928); M. C. Gutzwiller, Chaos in classical and quantum mechanics. (Springer, New York, 1990). W. H. Miller, J. Chem. Phys. 53, 3578 (1970). W. H. Miller, Journal of Physical Chemistry A 105 (13), 2942 (2001). W. H. Miller, Journal of Chemical Physics 95 (12), 9428 (1991); E. J. Heller, Journal of Chemical Physics 95 (12), 9431 (1991); E. J. Heller, Journal of Chemical Physics 94 (4), 2723 (1991); K. G. Kay, J. Chem. Phys. 100, 4377 (1994); K. G. Kay, J. Chem. Phys. 100, 4432 (1994).

M. F. Herman and E. Kluk, Chem. Phys. 91, 27 (1984).

R. Gelabert, X. Gimenez, M. Thoss, H. B. Wang, and W. H. Miller, Journal of Chemical Physics 114 (6), 2572 (2001); X. Sun, H. B. Wang, and W. H. Miller, Journal of Chemical Physics 109 (17), 7064 (1998); H. Wang, X. Song, D. Chandler, and W. H. Miller, J. Chem. Phys. 110, 4828 (1999); H. Wang, M. Thoss, K. L. Sorge, R. Gelabert, X. Gimenez, and W. H. Miller, J. Chem. Phys. 114, 2562 (2001); M. L. Brewer, J. S. Hulme, and D. E. Manolopoulos, J. Chem. Phys. 106, 4832 (1997); F. Grossmann, Phys. Rev. A 60, 1791 (1999); N. J. Wright and N. Makri, J. Phys. Chem. B 108, 6816 (2004); A. Nakayama and N. Makri, Proc. Nat. Acad Sci. USA 102, $4230(2005)$.

X. Sun and W. H. Miller, Abstracts of Papers of the American Chemical Society 213, 94 (1997). J. A. Poulsen, G. Nyman, and P. J. Rossky, Journal of Physical Chemistry B 108 (51), 19799 (2004).

N. J. Wright and N. Makri, Journal of Chemical Physics 119 (3), 1634 (2003).

A. Nakayama and N. Makri, J. Chem. Phys. 119, 8592 (2003).

J. Liu, A. Nakayama, and N. Makri, Molecular Physics 104 (8), 1267 (2006).

A. R. Walton and D. E. Manolopoulos, Mol. Phys. 87, 961 (1996); H. B. Wang, D. E. Manolopoulos, and W. H. Miller, Journal of Chemical Physics 115 (14), 6317 (2001); X. Sun and W. H. Miller, Journal of Chemical Physics 110 (14), 6635 (1999); K. Thompson and N. Makri, Phys. Rev. E 59, R4729 (1999); K. Thompson and N. Makri, J. Chem. Phys. 110, 1343 (1999).

N. Makri and K. Thompson, Chem. Phys. Lett. 291, 101 (1998).

Q. Shi and E. Geva, J. Phys. Chem. A 107 (9070-9078) (2003).

S. Bonella, D. Montemayor, and D. F. Coker, Proceedings of the National Academy of Sciences of the United States of America 102 (19), 6715 (2005).

P. A. Frantsuzov and V. A. Mandelshtam, Journal of Chemical Physics 121 (19), 9247 (2004).

P. Frantsuzov, A. Neumaier, and V. A. Mandelshtam, Chemical Physics Letters 381 (1-2), 117 (2003).

E. J. Heller, Chem. Phys. Lett. 34, 321 (1975); E. J. Heller, J. Chem. Phys. 64, 63 (1976).

B. Hellsing, S. I. Sawada, and H. Metiu, Chemical Physics Letters 122 (4), 303 (1985).

J. Shao and E. Pollak, (submitted).

N. Makri and W. H. Miller, Journal of Chemical Physics 116 (21), 9207 (2002).

Y. Zhao and W. H. Miller, Journal of Chemical Physics 117 (21), 9605 (2002).

B. J. Berne and G. D. Harp, Adv. Chem. Phys. 17, 63 (1970). 
W. H. Miller, S. D. Schwartz, and J. W. Tromp, Journal of Chemical Physics 79 (10), 4889 (1983).

W. H. Miller, Adv. Chem. Phys. 25, 69 (1974); W. H. Miller, Proceedings of the National Academy of Sciences of the United States of America 102 (19), 6660 (2005).

33 W. H. Miller, (submitted).

34 P. A. Frantsuzov, D. Meluzzi, and V. A. Mandelshtam, Physical Review Letters 96 (11) (2006); P. A. Frantsuzov and V. A. Mandelshtam, Physical Review E 72 (3) (2005).

D. C. Marinica, M. P. Gaigeot, and D. Borgis, Chemical Physics Letters 423 (4-6), 390 (2006).

E. Jezek and N. Makri, J. Phys. Chem. 105, 2851 (2001); M. Thoss, H. B. Wang, and W. H. Miller, Journal of Chemical Physics 114 (21), 9220 (2001).

37 K. Thompson and N. Makri, Physical Review E 59 (5), R4729 (1999).

38 J. Liu and N. Makri, Chemical Physics 322 (1-2), 23 (2006).

39 Thermodynamic Properties of Neon, Argon, Krypton, and Xenon, edited by V. A. Rabinovich, A. A. Vasserman, V. I. Nedostup, and L. S. Veksler (Springer-Verlag, Berlin, 1988); J. K. Johnson, J. A. Zollweg, and K. E. Gubbins, Molecular Physics 78 (3), 591 (1993).

K. Imre, E. Ozizmir, Rosenbau.M, and P. F. Zweifel, Journal of Mathematical Physics 8 (5), 1097 (1967); E. Pollak and J. L. Liao, Journal of Chemical Physics 108 (7), 2733 (1998).

$44 \quad$ N. Makri, Journal of Physical Chemistry B 106 (33), 8390 (2002).

45 R. Kubo, M. Toda, and N. Hashitsume, Statistical Physics, 2nd ed. (Springer-Verlag, Heidelberg, 1991).

W. H. Miller, Y. Zhao, M. Ceotto, and S. Yang, Journal of Chemical Physics 119 (3), 1329 (2003). 